When gap solitons become embedded solitons: a generic unfolding

Wagenknecht, T. and Champneys, A. R.

2003

MIMS EPrint: 2006.389

Manchester Institute for Mathematical Sciences

School of Mathematics

The University of Manchester

\footnotetext{
Reports available from: http://eprints.maths.manchester.ac.uk/

And by contacting: The MIMS Secretary

School of Mathematics

The University of Manchester

Manchester, M13 9PL, UK
} 


\title{
When gap solitons become embedded solitons; a generic unfolding
}

\author{
T. Wagenknecht ${ }^{1}$ \\ Department of Mathematics, TU Ilmenau, D-98684 Ilmenau, Germany, \\ E-mail: thomas.wagenknecht@tu-ilmenau.de
}

A. R. Champneys

Department of Engineering Mathematics, University of Bristol, Bristol BS8 1TR, UK, E-Mail: a.r.champneys@bristol.ac.uk

\begin{abstract}
A two-parameter unfolding is considered of single-pulsed homoclinic orbits to an equilibrium with two real and two zero eigenvalues in fourth-order reversible dynamical systems. One parameter controls the linearisation, with a transition occurring between a saddle-centre and a hyperbolic equilibrium. In the saddle-centre region, the homoclinic orbit is of codimension-one, which is controlled by the second generic parameter, whereas when the equilibrium is hyperbolic the homoclinic orbit is structurally stable. A geometric approach reveals the homoclinic orbits to the saddle to be generically destroyed either by developing an algebraically decaying tail or through a fold, depending on the sign of the perturbation of the second parameter. Special cases of different actions of $\mathbb{Z}_{2}$-symmetry are considered, as is the case of the system being Hamiltonian. Application of these results is considered to the transition between embedded solitons (corresponding to the codimension-one homoclinic orbits) and gap solitons (the structurally stable ones) in nonlinear wave systems. The theory is shown to match numerical experiments on two models arising in nonlinear optics and on a form of 5th-order Korteweg de Vries equation.
\end{abstract}

Key words: embedded soliton, homoclinic bifurcation, degenerate equilibrium, reversible system

PACS: $02.30 . \mathrm{Hq}, 05.45-\mathrm{a}, 42.65 . \mathrm{Tg}$

$\overline{1}$ Corresponding author 


\section{Introduction}

\subsection{Overview}

There has been much interest in recent years in the existence of localised coherent structures in nonlinear media, especially in optics. A particularly important class of localised structure are solitary waves, or "solitons" to give them their physical name (although we do not assume complete integrability here). In situations governed by higher-order or multi-component partial differential equation (PDE) models in $1+1$ dimensions, the spectrum of linearised waves generally has at least two branches. If these branches do not fill out the entire possible spectrum of wave frequencies, then one has the possibility of a gap in the linear spectrum where exponentially localised solutions can exist, so called gap solitons [12]. Such solutions can be linearly stable solutions of the PDE in that they are attractors for a range of initial data. They are also typically structurally stable, in that they exist for a range of frequency and other parameter values.

In contrast, an embedded soliton (ES) is a solitary wave which exists despite having its internal frequency in resonance with linear waves. Specifically, they occur in a two-component model when the dispersion relation has only one branch. In contrast to gap solitons, embedded solitons are not structurally stable in that they exist as codimension-one solutions, i.e., at discrete values of the frequency [34,11]. In these parameter ranges quasi-solitons (or "generalized solitary waves" [1]) in the form of solitary waves with non-decaying periodic tails are generic. However, at some special values of the internal frequency, the amplitude of the tail may exactly vanish, giving rise to an isolated soliton embedded into the continuous spectrum. Another interesting feature of ESs is that they may at best be only linearly neutrally stable, being subject to a weak one-sided algebraic instability, see e.g. [34,28]. That is, if one makes an energy increasing perturbation then via the shedding of radiation, the initial condition relaxes algebraically back to the solitary wave. In contrast, energy decreasing perturbations cause the solitary wave to decay algebraically away. The existence of embedded solitons has been established in a number of physical models including generalised 5th-order Korteweg-de Vries (KdV) equations [20,4,6,33], coupled KdV equations [17], in nonlinear Schrödinger (NLS) equations with higher-order derivatives [3,16] and in various coupled NLS-type equations arising in nonlinear optics $[34,10,8]$.

Soliton solutions in each of these example PDEs reduce, via a travelling-wave or steady-state reduction to homoclinic orbits of ordinary differential equations (ODEs). These ODEs typically have the structure of being fourth-order, reversible and Hamiltonian. The parameter region that supports embedded 
solitons corresponds to when the linearisation of the origin (the trivial fixed point) in such an ODE system is a saddle-centre. That is, after diagonalising the system, one two-dimensional component gives rise to imaginary eigenvalues $\pm i \omega$ (corresponding to a continuous branch in the linear spectrum of the PDE system), and the other to real eigenvalues $\pm \lambda$ (corresponding to a gap in the linear spectrum). In contrast, the gap soliton parameter region is where the eigenvalues of the origin are purely real $\pm \lambda_{1}, \pm \lambda_{2}$.

Both single-humped (1-pulse) and multi-humped ( $N$-pulse) embedded solitons have been discovered. General theories explain how the multi-pulses accumulate on the parameter values at which the 1-pulses exist $[27,23]$. This paper concerns only the single humped variety, since to date all non-fundamental embedded solitons have been found to be (exponentially) unstable as solutions of the underlying PDEs, see e.g. [11,35].

The key question we wish to address is what happens as we trace a path of embedded solitons up to a parameter value $\omega=0$ at which it passes over into being a gap soliton. We shall not here consider the question of the stability of the solitary waves undergoing this process, merely how can a codimension-one object cross a boundary and become structurally stable? Or from the point of view of the gap solitons, how does a 1-parameter family of solitons become degenerate as it transforms into an isolated branch of embedded solitons? We shall study this question using dynamical systems theory applied to reversible systems.

The rest of this section introduces the mathematical setting. Section 2 then contains the main part of the paper, the presentation of the geometric approach that leads to an unfolding of the degenerate codimension-two situation at the heart of this paper. Section 3 goes on to analyse systems which additionally have a reflection symmetry, such as often arises in NLS systems. In Section 4 we consider the important class of systems that are additionally Hamiltonian and show that in this case the previously obtained results are valid for symmetric solutions. Finally, Section 5 performs numerical computations on three example systems that have been previously shown to support the embedded-soliton to gap-soliton transition, and reveals excellent agreement with the preceding theory.

\subsection{The mathematical setting}

We consider four-dimensional ODEs

$$
\dot{x}=f(x, \lambda), \quad x \in \mathbb{R}^{4}, \lambda \in \mathbb{R}^{l}
$$

and assume that 
(H1) There exists a (linear) involution $R: \mathbb{R}^{4} \rightarrow \mathbb{R}^{4}$ such that

$$
f(R x, \lambda)+R f(x, \lambda)=0, \quad \forall x, \lambda .
$$

Moreover with Fix $(R):=\{x: R x=x\}$ we have $\operatorname{dim}(\operatorname{Fix}(R))=2$.

So, we are concerned with reversible systems. A fundamental characteristic of such systems is that with $x(t)$ also $R x(-t)$ is a solution. If $x(t)=R x(-t)$, that is, if for the corresponding orbit $X(t):=\{x(t): t \in \mathbb{R}\}$ we have $R X=X$ then we call the solution or the orbit symmetric. As general references concerning the theory of reversible systems we refer to $[5,13,31]$.

Turning to equilibria of (1) we assume that the origin is an equilibrium of (1) which is non-hyperbolic for $\lambda=0$, that is

(H2) $f(0, \lambda)=0 \forall \lambda$, and $\sigma\left(D_{1} f(0,0)\right)=\{0\} \cup\{ \pm \mu\}$ with 0 being a non-semisimple eigenvalue and $\mu \in \mathbb{R}^{+}$.

According to (H2) the origin is a symmetric equilibrium of (1). In particular, the linearisation $D_{1} f(0, \lambda)$ is a reversible matrix and thus if $\mu$ is an eigenvalue then so is $-\mu$. This observation shows that assumption (H2) is generically met in one-parameter families of reversible ODEs.

Our final assumption concerns the existence of a homoclinic orbit $\Gamma$.

(H3) At $\lambda=0$ equation (1) possesses an orbit $\Gamma=\{\gamma(t): t \in \mathbb{R}\}$ homoclinic to 0, i.e. $\gamma(t) \rightarrow 0$ as $t \rightarrow \pm \infty$. Furthermore, $R \Gamma=\Gamma$ and choosing $\alpha$ such that $0<\alpha<\mu$ we have $\|\gamma(t)\| e^{\alpha t} \rightarrow 0$ as $t \rightarrow \infty$.

The last part of (H3) merely ensures that $\Gamma$ is contained in the intersection of the stable and unstable manifold of 0 . Homoclinic orbits with such an exponential bound will play a distinguished role in the forthcoming analysis and we will call them fast decaying. We study bifurcations of homoclinic orbits from the primary orbit $\Gamma$. Thereby we concentrate on the existence of onehomoclinic orbits (both fast and slowly decaying) to the origin, that is orbits which are contained in a tubular neighbourhood of $\Gamma$ and make exactly one winding.

The main idea of our analysis is the following. Using the Centre Manifold Theorem [18] we can describe the local bifurcation of the equilibrium in a family of reversible vector fields on the "centre manifolds" $W_{\lambda}^{c}$. Points of intersection of the corresponding "centre-stable" and "centre-unstable manifolds" $W_{\lambda}^{c s(c u)}$ then correspond to homoclinic orbits to $W_{\lambda}^{c}$. (Using these notions for the manifolds is a slight abuse of language; see Section 2.1 for their construction.) 
Precise information about the asymptotic behaviour of the corresponding homoclinic orbits is obtained by using the invariant foliation of $W_{\lambda}^{c s}$ and performing a projection along stable fibres. In this way we derive a bifurcation diagram for one-homoclinic orbits to the origin.

The next part of the analysis concerns systems of equations which are additionally $\mathbb{Z}_{2}$-symmetric. Depending on the action of the symmetry on the homoclinic orbit $\Gamma$ and on the centre manifold we have to distinguish several bifurcation scenarios. However, most of the results can immediately be derived from the preceding analysis when no $\mathbb{Z}_{2}$-symmetry was present. We finally turn to systems being both reversible and Hamiltonian and discuss the bifurcation of symmetric orbits from $\Gamma$.

In the present paper we have chosen to perform an analysis that consists of purely geometrical considerations. We remark that there exists a supplementary functional-analytic approach which is based on Lin's method, [26,29]. This method was originally developed for the treatment of connecting orbits between hyperbolic equilibria of ODEs. A generalization to the case of homoclinic orbits to non-hyperbolic equilibria can be found in [21,32]. Using this technique one can derive bifurcation equations for fast decaying homoclinic orbits and for the intersection of $W_{\lambda}^{c s} \cap W_{\lambda}^{c u}$.

\section{Bifurcation of one-homoclinic orbits in the general case}

\subsection{Bifurcation of the equilibrium}

Let us start by describing the bifurcation of 0 in a suitable way. For this purpose we consider the extended system

$$
\begin{aligned}
& \dot{x}=f(x, \lambda) \\
& \dot{\lambda}=0
\end{aligned}
$$

with an equilibrium $(x, \lambda)=(0,0)$ possessing an $l+2$-dimensional centre manifold $\mathfrak{W}^{c}$ and $l+3$-dimensional centre-(un)stable manifolds $\mathfrak{W}^{c s(c u)}$, because of (H2). Each of these manifolds is foliated into $\{\lambda=$ const. $\}$-slices which we will denote by $W_{\lambda}^{c}$ and $W_{\lambda}^{c s(c u)}$, respectively. The manifolds are not unique in general and in accordance with the reversibility we can choose $W_{\lambda}^{c s}=R W_{\lambda}^{c u}$ and set $W_{\lambda}^{c}=W_{\lambda}^{c s} \cap W_{\lambda}^{c u}$. By the Centre Manifold Theorem, see $[18,19]$, and since $R W_{\lambda}^{c}=W_{\lambda}^{c}$ we can thus follow the evolution of small bifurcating solutions of (1) in a family of reversible vector fields on $W_{\lambda}^{c}$. We note that we can extend the local manifolds $W_{\lambda}^{c s(c u)}$ along the orbit $\Gamma$ to derive global centre-(un)stable 
manifolds. We will denote these global manifolds by the same symbols.

Since $\operatorname{dim}\left(W_{\lambda}^{c}\right)=2$, i.e. we deal with planar vector fields, the equilibrium is completely described by its normal form, see [15,25]. Introducing $\left(y_{1}, y_{2}\right)$ coordinates in $W_{\lambda}^{c}$ the involution $R$ can be assumed to act as $R:\left(y_{1}, y_{2}\right) \mapsto$ $\left(y_{1},-y_{2}\right)$ and a reversible normal form is given in [25] by

$$
\begin{aligned}
& \dot{y}_{1}=y_{2} \\
& \dot{y}_{2}=\sum_{k \geq 1} a_{k}(\lambda) y_{1}^{k} .
\end{aligned}
$$

Note that by $(\mathrm{H} 2)$ we have $a_{1}(0)=0$ and considering the generic case $a_{2}(0) \neq 0$ we can with no loss of generality assume that the normal form for our problem reads

$$
\begin{aligned}
& \dot{y}_{1}=y_{2} \\
& \dot{y}_{2}=\lambda_{1} y_{1}+y_{1}^{2} .
\end{aligned}
$$

Being Hamiltonian the system (2) is easily analysed. We find the familiar phase portrait of the reversible transcritical bifurcation, see Figure 1 . The origin is a centre for $\lambda_{1}<0$ and a saddle for $\lambda_{1}>0$. In addition, for $\lambda_{1}>0$ it is connected to itself by a (small) homoclinic orbit $\Upsilon$.
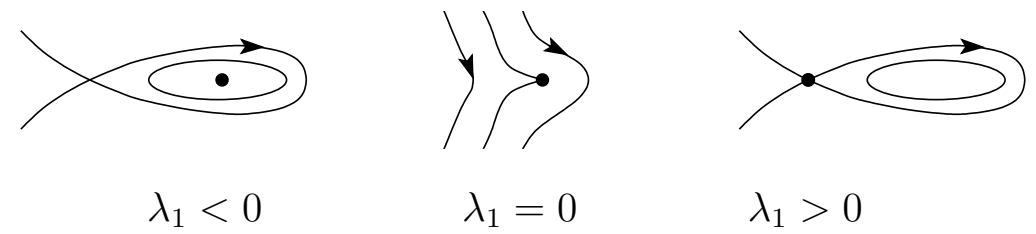

Figure 1. Phase portrait for the normal form (2) of the reversible transcritical bifurcation in $W_{\lambda}^{c}$. The ' $\bullet$ ' denotes the equilibrium 0 .

\subsection{Detection of one-homoclinic orbits}

In this central part of the paper we will investigate how homoclinic orbits to the origin bifurcate from the primary orbit $\Gamma$. This will proceed in two steps. First we introduce some cross-section $\Sigma$ to $\Gamma$ and study the intersection of $W_{\lambda}^{c s}$ and $W_{\lambda}^{c u}$ in $\Sigma$. (Note that we refer to the global centre-stable and centreunstable manifold here.) In this way we compute homoclinic orbits to $W_{\lambda}^{c}$. Of course, $W_{\lambda}^{c s(c u)}$ may intersect $\Sigma$ many times . But since we are interested in onehomoclinic solutions our analysis concerns only those pieces of the manifolds that visit $\Sigma$ for the first time. In a second step we analyse the asymptotic behaviour of these homoclinic orbits in detail by performing a projection along stable fibres. This method allows us to determine all homoclinic orbits to the 


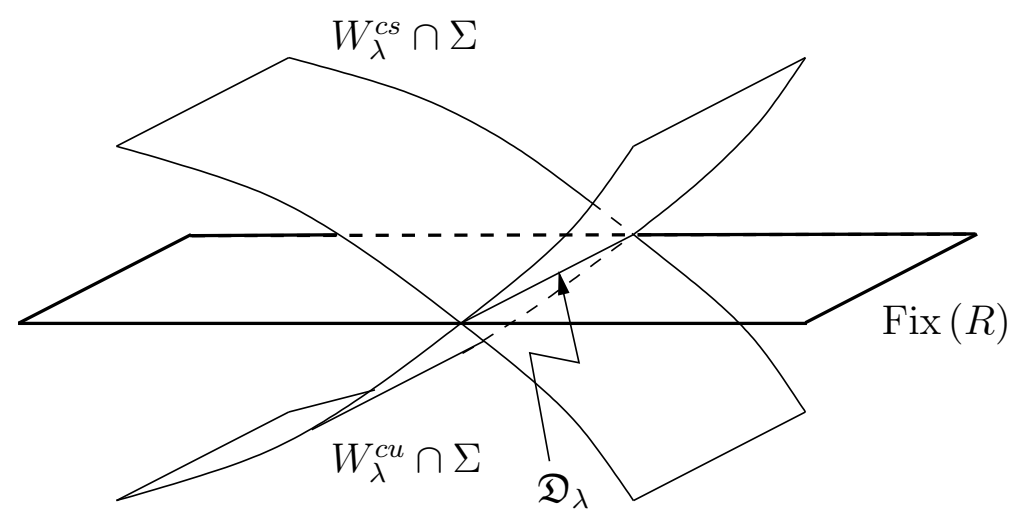

Figure 2. Position of the traces of $W_{\lambda}^{c s(c u)}$ in $\Sigma$ under assumption (H4)

origin. Fast decaying homoclinic orbits will play a distinguished role here. A discussion of their bifurcation reveals the problem to be of codimension two.

We first remark that since $\Gamma$ is symmetric we have $\Gamma \cap \operatorname{Fix}(R) \neq \emptyset$. Thus, we can choose $\gamma(0) \in \operatorname{Fix}(R)$ and we will introduce a cross-section $\Sigma$ to $\Gamma$ at $\gamma(0)$

$$
\Sigma=\gamma(0)+Z
$$

where we can choose the space $Z$ such that $R Z=Z$. This implies in particular that $\operatorname{Fix}(R) \subset Z$. The following transversality condition is crucial for our analysis.

(H4) At $\gamma(0)$ we have $W_{\lambda=0}^{c s} \pitchfork W_{\lambda=0}^{c u}$.

We claim that from (H4) we can gather all the information we need. First note that by standard arguments the transverse intersection of the manifolds will persist for $\lambda$ sufficiently close to 0 . Now, the traces of $W_{\lambda}^{c s}$ and $W_{\lambda}^{c u}$ in $\Sigma$ are both two-dimensional. Their transverse intersection will therefore be a one-dimensional object, i.e. some curve $\mathfrak{D}_{\lambda}$ in $\Sigma$. Furthermore, assumption (H4) implies that the traces of both $W_{\lambda=0}^{c s}$ and $W_{\lambda=0}^{c u}$ intersect Fix $(R)$ transversally in $\Sigma$. Indeed, assuming this was not the case would amount to $\left(T_{\gamma(0)} W_{\lambda=0}^{c s} \cap Z\right) \subset \operatorname{Fix}(R)$. But because of $R W_{\lambda}^{c s}=W_{\lambda}^{c u}$ this would yield $T_{\gamma(0)} W_{\lambda=0}^{c s}=T_{\gamma(0)} W_{\lambda=0}^{c u}$ in contradiction to (H4). Repeating the arguments above we see that both $W_{\lambda}^{c s}$ and $W_{\lambda}^{c u}$ intersect Fix $(R)$ in $\Sigma$ in some curve. Hence, $\mathfrak{D}_{\lambda} \subset \operatorname{Fix}(R)$, see Figure 2 for an illustration. We formulate this result as a first lemma.

Lemma 2.1 Under assumptions (H1)-(H4) equation (1) possesses a one-parameter family of symmetric homoclinic orbits to the manifolds $W_{\lambda}^{c}$ for $|\lambda|$ sufficiently small. This family of orbits intersects $\Sigma$ in a smooth curve $\mathfrak{D}_{\lambda} \subset$ Fix $(R)$. 


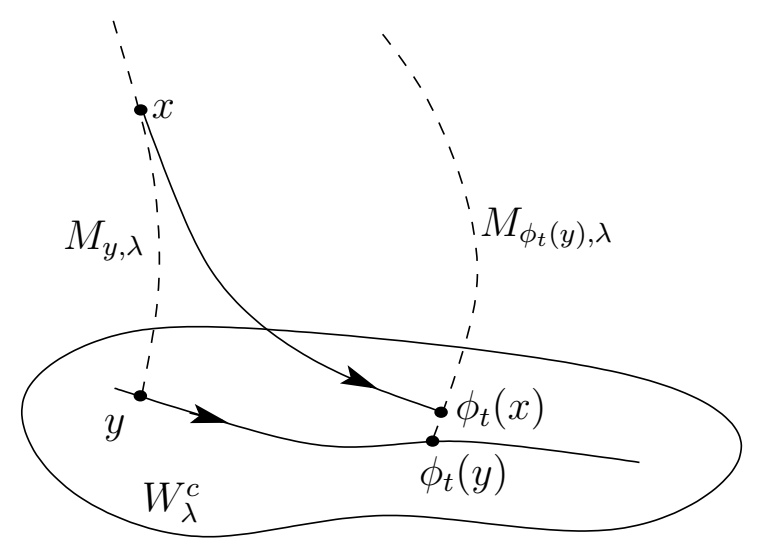

Figure 3. Invariance of the stable fibres: The orbit through $x \in M_{y, \lambda}$ stays in the fibre with base point $\phi_{t}(y)$ for all $t \geq 0$.

Because of assumption (H4) the set of homoclinic orbits to $W_{\lambda}^{c}$ does not depend on $\lambda$. But we are interested in homoclinic orbits to the origin. It thus remains to find out which of the orbits in Lemma 2.1 are asymptotic to the origin. Because of the symmetry of the orbits it suffices to study their behaviour as $t \rightarrow \infty$. For this we make use of the invariant foliation of $W_{\lambda}^{c s}$ and perform a projection along stable fibres. A distinguished role will be played by the stable fibre of the origin, which is related to the existence of fast decaying homoclinic orbits, that is, orbits which satisfy an exponential bound as in (H3). Dealing with such orbits we find that our unfolding requires a second parameter.

Let us introduce the technique. We can think of $W_{\lambda}^{c s}$ as being foliated into stable fibres. That is, from each point $y \in W_{\lambda}^{c}$ there originates a one-dimensional manifold $M_{y, \lambda} \subset W_{\lambda}^{c}$ such that

$$
W_{\lambda}^{c s}=\bigcup_{y \in W_{\lambda}^{c}} M_{y, \lambda}
$$

It will be of importance for us that the fibres form an invariant foliation in the following sense: Let $\phi_{t}(x)$ denote the solution of (1) which starts in $x$ at $t=0$. Then we have for the fibres that

$$
\phi_{t}\left(M_{y, \lambda}\right) \subset M_{\phi_{t}(y), \lambda} .
$$

Geometrically, (3) means that points in $M_{y, \lambda}$ follow the trajectory of the base point under the flow, see also Figure 3. Of particular interest will be the case when the base point approaches the origin itself. In this case (3) implies that all points in $M_{y, \lambda}$ will do so, too.

We first deal with the stable fibre $M_{0, \lambda}$ of the origin. It is easy to see that a fast decaying homoclinic orbit exists if and only if $M_{0, \lambda}$ intersects $W_{\lambda}^{c u}$ (or, equivalently, if it intersects Fix $(R))$ in $\Sigma$. Indeed, the orbit is homoclinic to 0 
because of its symmetry established in Lemma 2.1. Furthermore, by (3) the fibre $M_{0, \lambda}$ itself is invariant. Therefore, an orbit that is contained in the fibre satisfies the exponential bound in (H3). In order to consider a generic situation for the bifurcation of fast decaying orbits we impose a transversality condition with regard to the stable fibre $M_{0, \lambda}$. For this define

$$
\mathfrak{M}_{0}:=\bigcup_{|\lambda| \text { small }} M_{0, \lambda}, \quad \mathfrak{W}^{c u}:=\bigcup_{|\lambda| \text { small }} W_{\lambda}^{c u} .
$$

(Note that $\mathfrak{W}^{c u}$ is nothing but the extended version of the local manifold introduced in Section 2.1.) We demand that

(H5) $\mathfrak{M}_{0} \pitchfork \mathfrak{W}^{c u}$ in $\gamma(0)$ at $\lambda=0$

Let us discuss the consequences of (H6). We can consider the intersection of $\mathfrak{M}_{0}$ and $\mathfrak{W}^{c u}$ in $\Sigma \times \mathbb{R}^{l}$ (recall that $\lambda \in \mathbb{R}^{l}$ in (1)). By counting dimensions one easily sees that (H5) implies this intersection to be $(l-1)$-dimensional. Thus, we can consider (1) with parameters $\left(\lambda_{1}, \lambda_{2}\right) \in \mathbb{R}^{2}$. In a suitable unfolding fast decaying homoclinic orbits then exist at a curve in parameter space. With no loss of generality we can assume this curve to be the $\lambda_{1}$-axis.

Lemma 2.2 Under assumption (H5) we can choose parameters $\left(\lambda_{1}, \lambda_{2}\right)$ such that the local bifurcation of the 0-equilibrium is described by (2) and such that (1) possesses a fast decaying homoclinic orbit if and only if $\lambda_{2}=0$.

Remark. The fibre $M_{0, \lambda}$ has a precise geometric meaning. For $\lambda_{1} \leq 0$, where 0 is a non-hyperbolic equilibrium, it is the stable manifold of this equilibrium. For $\lambda_{1}>0$ it is the strong-stable manifold of 0 .

In order to investigate whether additional homoclinic orbits to 0 exist we project the curve $\mathfrak{D}_{\lambda}$ along stable fibres from $\Sigma$ to $W_{\lambda}^{c}$. Since the fibres depend smoothly on both their base point $y \in W_{\lambda}^{c}$ and on the parameter $\lambda$ this projection is a smooth map. It is furthermore injective, since the fibre $M_{0,0}$ is just the homoclinic orbit $\Gamma$ which intersects $\Sigma$ transversally. Therefore, all fibres $M_{y, \lambda}$ will do the same for $\lambda$ small. Thus, the image of $\mathfrak{D}_{\lambda}$ under the projection is some curve $\mathfrak{C}_{\lambda}$ which intersects the origin for $\lambda=0$. We impose the following transversality condition upon this curve.

(H6) $\quad \mathfrak{C}_{\lambda=0} \pitchfork \operatorname{Fix}(R)$

So we demand that at $\lambda=0$ the curve is not tangent to $\operatorname{Fix}(R)$. This is essentially an assumption concerning the geometry within $W_{\lambda=0}^{c s}$.

In consequence we obtain the bifurcation diagram in Figure 4. This diagram 


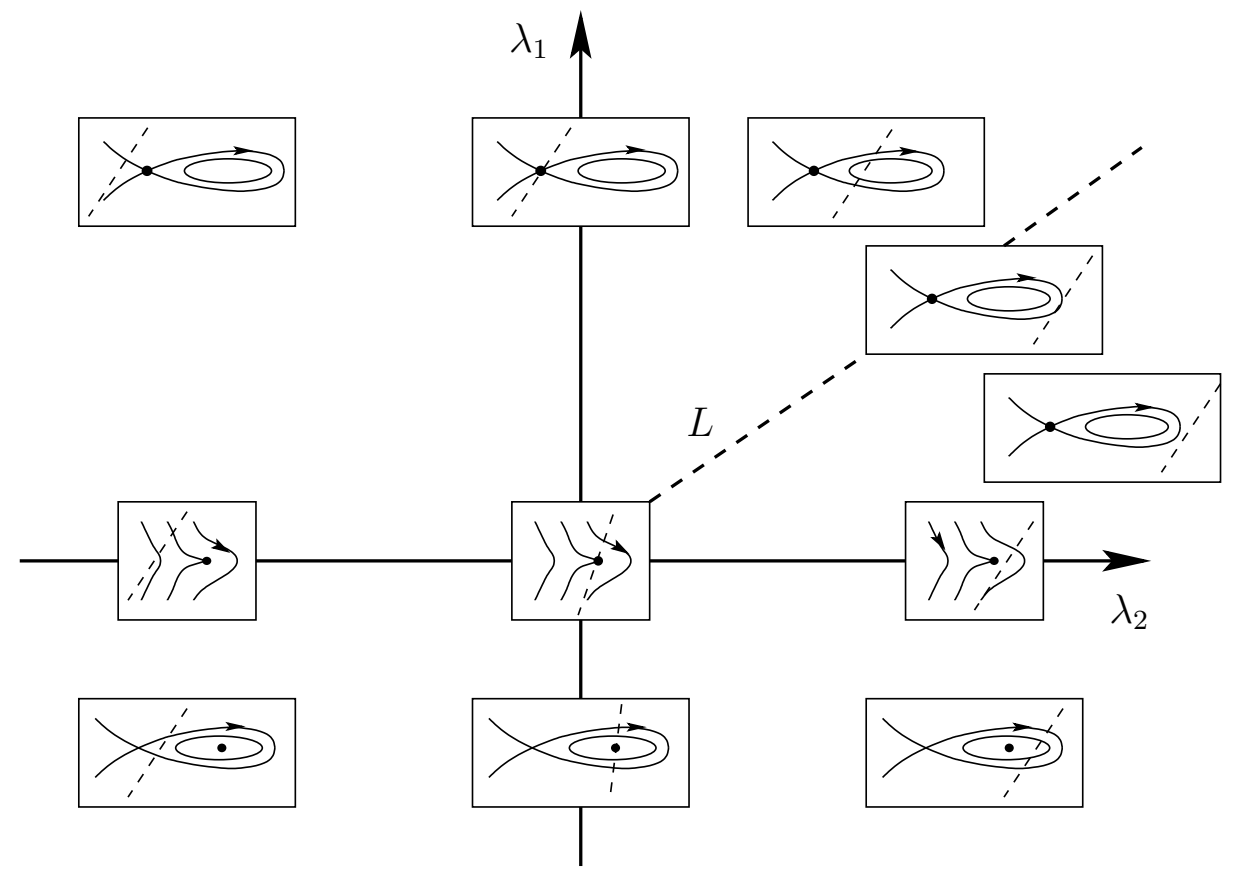

Figure 4. Bifurcation diagram for one-homoclinic orbits near $\Gamma$. The dashed line in each small box shows the curve $\mathfrak{C}_{\lambda}$.

shows the local behaviour in $W_{\lambda}^{c}$ together with the curve $\mathfrak{C}_{\lambda}$ in dependence of the parameters. We recall that the parameter $\lambda_{1}$ controls the local bifurcation of 0 . The parameter $\lambda_{2}$ has been introduced to unfold the bifurcation of fast decaying homoclinic orbits. Such orbits exist if $\mathfrak{C}_{\lambda}$ intersects the origin. Hence, $\lambda_{2}$ controls the position of $\mathfrak{C}_{\lambda}$ in $W_{\lambda}^{c}$. Since the stable fibres depend smoothly on $\lambda$ and because of (H5), the curve $\mathfrak{C}_{\lambda}$ moves linearly to lowest order with respect to $\lambda_{2}$.

Let us discuss the diagram. We first consider the situation for $\lambda_{1}<0$. Here, 0 is a saddle-centre and we find that $\mathfrak{C}_{\lambda}$ intersects the origin if and only if $\lambda_{2}=0$. As observed above this intersection corresponds to a fast decaying homoclinic orbit. For $\lambda_{2} \neq 0$ the curve $\mathfrak{C}_{\lambda}$ only intersects periodic orbits near 0 . Using the invariance property (3) and the symmetry it is easy to see that each of those intersection points yields a homoclinic orbit to a periodic orbit. However, such orbits are not of particular interest to us. We conclude that for $\lambda_{2} \neq 0$ no homoclinic orbits to the origin exist. This result is also compatible with the fact that symmetric homoclinic orbits to a saddle-centre are of codimension one in the class of reversible systems.

For $\lambda_{1}=0$ we find homoclinic orbits when $\lambda_{2} \leq 0$. In fact, for $\lambda_{2}=0$ this is implied by assumption (H2), while for $\lambda_{2}<0$ we see that $\mathfrak{C}_{\lambda}$ intersects the curve of points which are asymptotic to the origin within $W_{\lambda}^{c}$ as $t \rightarrow \infty$. Therefore the corresponding orbit which starts in $\Sigma$ will do so as well by (3), and moreover, being symmetric, it will approach $R 0=0$ as $t \rightarrow-\infty$. So, it 
is a homoclinic orbit, which in addition decays algebraically. An elementary calculation for the normal form (2) shows that the corresponding solution will eventually decay like $1 / t^{2}$ for $t \rightarrow \pm \infty$.

Let us now discuss the bifurcations when $\lambda_{1}>0$. Here the origin is a real saddle and for $\lambda_{2}=0$ there exists a fast decaying homoclinic orbit to this saddle. These orbits lie in the strong (un)stable manifold of 0 and one would expect a reversible orbit-flip bifurcation when the parameters are varied. Indeed, we find this in Figure 4. For $\lambda_{2}<0$ the curve $\mathfrak{C}_{\lambda}$ intersects the stable manifold of 0 (in $W_{\lambda}^{c}$ ) which shows the existence of a slowly decaying homoclinic orbit. For $\lambda_{2}>0$ sufficiently small we actually find two points of intersection of $\mathfrak{C}_{\lambda}$ with the small homoclinic orbit $\Upsilon$ in $W_{\lambda}^{c}$. (Note that this is a consequence of (H6).) Therefore two slowly decaying homoclinic orbits to the origin exist. However, a closer examination shows that the upper point of intersection corresponds to an orbit which first follows $\Gamma$ for some time, but when the orbit is close to the centre manifold it does not approach the 0-equilibrium directly but runs along $\Upsilon$. We will therefore not view this orbit as a one-homoclinic orbit to 0 since it comprises a gluing between $\Gamma$ and $\Upsilon$. Doing so, we find agreement with general results concerning the reversible orbit-flip bifurcation derived in [30] which show that generically only a single one-homoclinic orbit exists.

Finally, another interesting bifurcation occurs when $\lambda_{2}$ is increased further. We find a curve $L$ on which the two points of intersection of $\mathfrak{C}_{\lambda}$ with $\Upsilon$ merge and vanish. This scenario corresponds to a saddle-node bifurcation of symmetric homoclinic orbits as was analysed in $[2,22]$. Such a bifurcation occurs when a homoclinic orbit becomes degenerate, i.e. when the tangent spaces of the stable and unstable manifold of 0 have another common direction along the orbit (in the four-dimensional case they thus agree). For $\lambda_{2}$ large enough there exists no homoclinic orbit to 0 . Let us give some explanation concerning the properties of the curve $L$ : First of all we recall that the motion of $\mathfrak{C}$ in $W_{\lambda}^{c}$ is linear in $\lambda_{2}$. On the other hand, it is an easy calculation using the normal form (2) that the size of the small homoclinic orbit $\Upsilon$ varies linear in $\lambda_{1}$. Therefore, we conclude that $L$ is the graph of some function $\lambda_{1}=a \lambda_{2}+o\left(\lambda_{2}\right)$ with $a>0, \lambda_{2} \geq 0$. We summarise the results in a theorem.

Theorem 2.3 Consider (1) under the assumptions (H1)-(H6) with parameters $\lambda=\left(\lambda_{1}, \lambda_{2}\right)$ chosen in accordance with the normal form (2) and with Lemma 2.2. Then fast decaying homoclinic orbits to 0 exist if and only if $\lambda_{2}=0$. For $\lambda_{1}<0$ no other one-homoclinic orbits exist.

In the case $\lambda_{1}=0$ we find one homoclinic orbit to the 0 -equilibrium if $\lambda_{2} \leq 0$ which is algebraically decaying for $\lambda_{2}<0$.

For $\lambda_{1}>0, \lambda_{2} \leq 0$ we find one homoclinic orbit to the 0 -equilibrium. For $\lambda_{2}>$ 
0 there exist two homoclinic orbits which coalesce in a saddle-node bifurcation on some curve $L:=\left\{\left(\lambda_{2}, \lambda_{1}\right): \lambda_{2} \geq 0, \lambda_{1}=a \lambda_{2}+o\left(\lambda_{2}\right)\right\}$, with some $a>0$.

Remark. We have restricted the analysis to homoclinic orbits to the origin merely because of the physical background we have in mind. Obviously, our method can also be used for a classification of the other homoclinic orbits found in Lemma 2.1. For example, using the invariance property (3) one immediately sees that an intersection of $\mathfrak{C}_{\lambda}$ with a periodic orbit in $W_{\lambda}^{c}$ corresponds to a homoclinic orbits to this periodic orbit. As a consequence we see that when $\lambda_{1}<0$ and $\lambda_{2}=0$ every periodic orbit in $W_{\lambda}^{c}$ is connected to itself by two homoclinic orbits. For $\lambda_{2} \neq 0$ there exists a critical amplitude $A$, depending linearly on $\lambda_{2}$, such that we only find homoclinic orbits to periodic orbits with an amplitude greater than $A$. Similarly, one can obtain a complete description of homoclinic orbits bifurcating from $\Gamma$.

\section{Cases with $\mathbb{Z}_{2}$-symmetry}

In this section we will consider the situation under the additional assumption that (1) is $\mathbb{Z}_{2}$-symmetric with respect to some involution $S$ (commuting with $R$ ), that is we have

$$
S f(x, \lambda)=f(S x, \lambda), \quad \forall(x, \lambda) .
$$

Since the composition $Q:=R \circ S$ gives another reversibility, this is equivalent to considering systems that are reversible with respect to two involutions. (This observation was formalised in the notion of a reversing symmetry group in [25].) An immediate consequence from (4) is that Fix $(S)$ is an invariant subspace for (1). We will see that we have to distinguish several cases according to the location of the subspace.

\subsection{Odd symmetry}

We will deal with (1) under (H1)-(H3) and assume additionally

(H7) $f$ is odd-symmetric, that is $f(x, \lambda)=-f(-x, \lambda)$ for all $(x, \lambda)$.

Equivalently we could have required $f$ to be reversible with respect to $Q:=$ $-R$, as well. We remark that since $S=-i d$ we have $\operatorname{Fix}(S)=\{0\}$.

Assumption (H7) has consequences for the local bifurcation of the equilibrium

0 . Now we have to consider systems (and therefore normal forms) in $W_{\lambda}^{c}$ that 


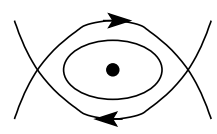

$\lambda_{1}<0$

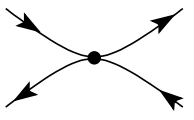

$\lambda_{1}=0$

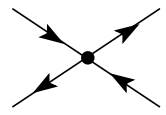

$\lambda_{1}>0$

Figure 5. Phase portrait for the normal form (5) of the reversible pitchfork bifurcation in $W_{\lambda}^{c}$. The ' $\bullet$ ' denotes the equilibrium at the origin.

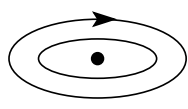

$\lambda_{1}<0$

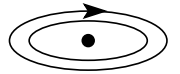

$\lambda_{1}=0$

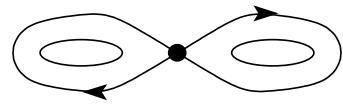

$\lambda_{1}>0$

Figure 6. Phase portrait for the normal form (6) of the reversible pitchfork bifurcation in $W_{\lambda}^{c}$. The '•' denotes the equilibrium 0 .

are reversible with respect to $R$ and $-R$. The corresponding general normal form from [25] then reads

$$
\begin{aligned}
& \dot{y}_{1}=y_{2} \\
& \dot{y}_{2}=\sum_{k \text { odd }} a_{k}(\lambda) y_{1}^{k},
\end{aligned}
$$

where we have again introduced $\left(y_{1}, y_{2}\right)$-coordinates in $W_{\lambda}^{c}$. (Note that we choose $R:\left(y_{1}, y_{2}\right) \rightarrow\left(y_{1},-y_{2}\right)$.) By (H2) we also have $a_{1}(0)=0$ and this time the generic situation is characterised by the assumption $a_{3}(0) \neq 0$ which leads to considering a reversible pitchfork bifurcation. Here we have to distinguish two qualitatively different cases. For $a_{3}(0)>0$ the normal form for the problem is given by

$$
\begin{aligned}
& \dot{y}_{1}=y_{2} \\
& \dot{y}_{2}=\lambda_{1} y_{1}+y_{1}^{3} .
\end{aligned}
$$

This corresponds to the so called 'eye-case' which leads to the emergence of a small heteroclinic cycle for $\lambda_{1}<0$. The phase portraits are presented in Figure 5 .

In the case $a_{3}(0)<0$ we deal with the normal form

$$
\begin{aligned}
& \dot{y}_{1}=y_{2} \\
& \dot{y}_{2}=\lambda_{1} y_{1}-y_{1}^{3} .
\end{aligned}
$$

This case is often referred to as the 'figure-eight' case since for $\lambda_{1}>0$ we find two homoclinic orbits $\Upsilon_{1,2}$ connecting the origin to itself, see Figure 6 for the phase portraits. 


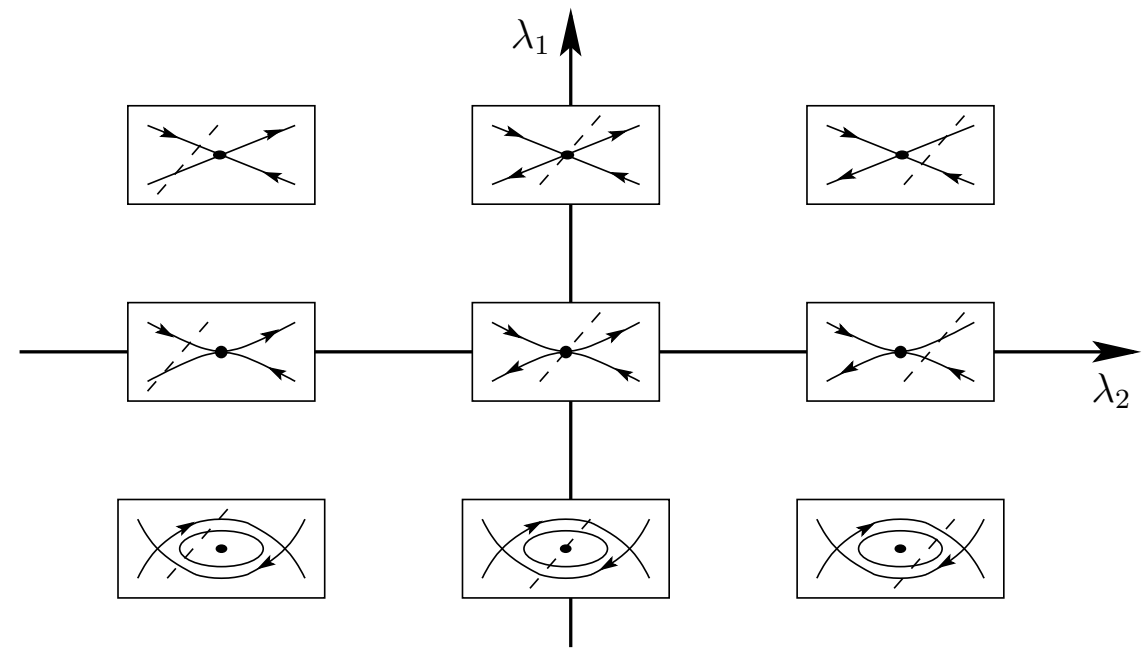

Figure 7. Bifurcation diagram for one-homoclinic orbits near $\Gamma$ in case of the normal form (5). The dashed line in each box shows the curve $\mathfrak{C}_{\lambda}$.

We can now proceed with the analysis of bifurcations from $\Gamma$ as in Section 2.2. But for that two things are important. First, $(H 7)$ implies that $-\Gamma$ is another fast decaying homoclinic orbit to the origin at $\lambda=0$. Second, we again impose (H4) which by symmetry also holds for points on $-\Gamma$. Thus, we can deal with the orbit $\Gamma$ alone and infer all results for $-\Gamma$ using the $\mathbb{Z}_{2}$-symmetry of (1).

Exclusively considering $\Gamma$ all the analysis of the last section can again be performed. Lemma 2.1 thus shows the existence of a manifold of homoclinic orbits to $W_{\lambda}^{c}$ of which all orbits are $R$-symmetric. Projecting along stable fibres in this case we also get a curve $\mathfrak{C}_{\lambda}$ for which the non-degeneracy condition (H6) is assumed to hold. Doing so, we derive two bifurcation scenarios, depending on which normal form describes the bifurcation of 0 . The diagrams are given in Figure 7 and 8. In the same manner as before we can turn the diagrams into a theorem.

Theorem 3.1 Consider (1) under the assumptions (H1)-(H7) with parameters $\lambda=\left(\lambda_{1}, \lambda_{2}\right)$ chosen in accordance with the normal form (5), (6) and Lemma 2.2. Then fast decaying homoclinic orbits to the origin near $\Gamma$ exist if and only if $\lambda_{2}=0$. For $\lambda_{1}<0$ no other one-homoclinic orbits exist.

In case the normal form for the local bifurcation is given by (5) then there exist additional homoclinic orbits for all $\lambda_{1} \geq 0, \lambda_{2} \neq 0$ which are algebraically decaying when $\lambda_{1}=0$.

When the local bifurcation is described by (6) then additional orbits only exist for $\lambda_{1}>0$. For $\left|\lambda_{2}\right|$ sufficiently small there exist two homoclinic orbits which 


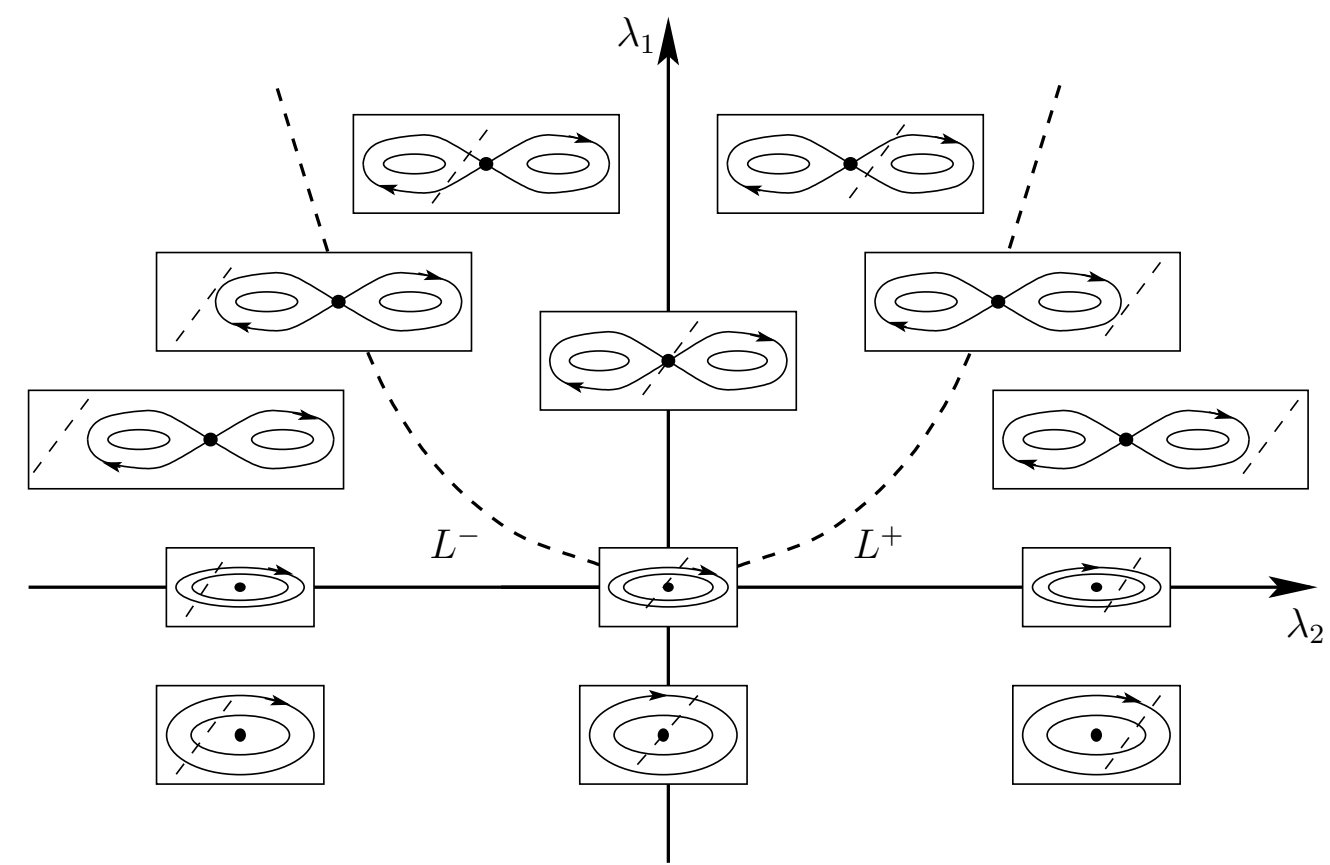

Figure 8. Bifurcation diagram for one-homoclinic orbits near $\Gamma$ in case of the normal form (6). The dashed line in each box shows the curve $\mathfrak{C}_{\lambda}$.

coalesce in a saddle-node bifurcation at some curve $L=L^{+} \cup L^{-}$given by $\lambda_{1}=a \lambda_{2}^{2}+o\left(\lambda_{2}^{2}\right)$, with some $a>0$.

By symmetry the assertions are also valid near $-\Gamma$.

Remark. For the figure-eight case we need again assumption (H6) to derive the bifurcation diagram. Furthermore, for the normal form (6) the size of the figure-eight depends quadratically on $\lambda_{1}$. More precisely, let $v_{1,2}:=( \pm v, 0)$ denote the two points of the figure-eight $\Upsilon_{1} \cup \Upsilon_{2}$ which have the largest respectively smallest $y_{1}$-component. Then we find that $v^{2}=2 \lambda_{1}$. This explains the shape of the 'saddle-node-curves' $L^{ \pm}$in Figure 8.

\section{2 $\mathbb{Z}_{2}$-symmetry with $W_{\lambda}^{c} \subset$ Fix $(S)$}

We are now interested in the case when the fixed space of $S$ is non-trivial. That is we consider (1) under (H1)-(H3) and assume in addition that

(H8) There exists an involution $S: \mathbb{R}^{4} \rightarrow \mathbb{R}^{4}$ commuting with $R$ and with $\operatorname{dim}(\operatorname{Fix}(S))=2$, such that $(4)$ is fulfilled.

As remarked before, the map $Q:=S \circ R$ then induces another reversing symmetry for (1). We remark that, within Fix $(S)$, the involutions $Q$ and $R$ 
agree.

With regard to our setup two situations have to be distinguished, depending on whether the symmetry affects the homoclinic orbit $\Gamma$ or the local bifurcation of the equilibrium $x=0$. We first deal with the latter case. Denoting the centre eigenspace of $D_{1} f(0,0)$ by $E^{c}$ let us assume that

(H9) $E^{c} \subset \operatorname{Fix}(S)$.

Under (H9) we can derive a bifurcation diagram for one-homoclinic orbits near $\Gamma$ in precisely the same manner as before. First, we see that within $W_{\lambda}^{c} \subset$ Fix $(S)$ the vector field is reversible with respect to one involution. Therefore the equilibrium 0 generically bifurcates in a transcritical bifurcation as in Section 2 above. The corresponding normal form for the local bifurcation is given by (2) and the local bifurcation diagram is as shown in Figure 1.

For the homoclinic orbit $\Gamma$ assumption (H9) yields that $Q \Gamma \neq \Gamma$, i.e. $\Gamma$ is not symmetric with respect to $Q$. Indeed, assuming that $\Gamma$ was $Q$-symmetric we would have $\gamma(0) \in \operatorname{Fix}(R) \cap \operatorname{Fix}(Q)$, i.e. $\gamma(0) \in \operatorname{Fix}(S)$. But this results in $\Gamma \subset \operatorname{Fix}(S)$, which is forbidden by (H9). We therefore conclude that $Q \Gamma$ is a second homoclinic orbit of (1).

Precisely as in Section 3.1 we can now consider each orbit separately and then immediately apply the results of Section 2.2. Thus the bifurcation diagram for one-homoclinic orbits in Figure 4 is valid for both $\Gamma$ and $Q \Gamma$. So, we obtain the next theorem.

Corollary 3.2 Consider (1) under assumptions (H1)-(H6), (H8), and (H9). Then $Q \Gamma$ is a second homoclinic orbit to 0 at $\lambda=0$ and Theorem 2.3 applies to both orbits $\Gamma$ and $Q \Gamma$.

Remark. A completely analogous result is valid when the local bifurcation of $x=0$ is described by the normal forms (5) or (6). This case is non-generic in our general setup. It could, however, arise when we assume an additional symmetry within the subspace Fix $(S)$. Then the bifurcation of one-homoclinic orbits from $\Gamma$ is described by Theorem 3.1.

\section{$3.3 \mathbb{Z}_{2}$-symmetry with $\Gamma \subset$ Fix $(S)$}

For the final case in this section we assume (H1)-(H3) and (H8) and in addition 
(H10) $Q \Gamma=\Gamma$.

Assumption (H10) implies that $\gamma(0) \in \operatorname{Fix}(R) \cap \operatorname{Fix}(Q)$, i.e. $\gamma(0) \in \operatorname{Fix}(S)$. So, by invariance of $\operatorname{Fix}(S)$ this means $\Gamma \subset \operatorname{Fix}(S)$. In particular, the stable and unstable manifold of 0 are contained in Fix $(S)$. Hence we conclude that $W_{\lambda}^{c} \cap \operatorname{Fix}(S)=\{0\}$. Within $W_{\lambda}^{c}$ we have to deal with vector fields that are reversible with respect to two involutions. That means, we are in the same situation as in Section 3.1, in that (5) or (6) give the suitable normal forms for the local bifurcation.

Consider (1) reduced to the invariant subspace Fix $(S)$. Then the system is also reversible and since $W_{\lambda}^{c} \cap \operatorname{Fix}(S)=\{0\}$ we find that the origin is a hyperbolic equilibrium for the reduced system. Thus, $\Gamma \subset \operatorname{Fix}(S)$ is a symmetric homoclinic orbit to a hyperbolic equilibrium. Therefore, the orbit is robust under reversible perturbations. In each one of the other cases, however, we find arbitrary small perturbations that destroy the fast decaying homoclinic orbit. We therefore conclude that the above analysis can not apply to the present scenario. In particular, no additional parameter is now required to control the existence of fast decaying homoclinic orbits. In the present situation our problem is of codimension one.

The main difference from the above cases is that under (H10) assumption (H4) is impossible. This is proved in [32] where the case was considered in detail. We only give a concise description of the results here and refer to that paper for a detailed discussion involving an analytical approach based on Lin's method. It is shown in [32] that (H10) gives rise to additional symmetries for $W_{\lambda}^{c s}$ and $W_{\lambda}^{c u}$ which imply that common directions in the tangent spaces of these manifolds are not allowed to lie in Fix $(S)$. We cite from [32]:

Lemma 3.3 Under (H10) the intersection of $W_{\lambda}^{c s}$ and $W_{\lambda}^{c u}$ is non-transverse, and moreover $W_{\lambda}^{c s(c u)} \pitchfork$ Fix $(R), W_{\lambda}^{c s(c u)} \pitchfork \operatorname{Fix}(Q)$.

From this lemma one can immediately conclude the existence of a manifold of $R$-symmetric homoclinic orbits and a manifold of $Q$-symmetric homoclinic orbits to $W_{\lambda}^{c}$ for all $\lambda$. A suitable non-degeneracy condition, see [32], excludes the existence of other one-homoclinic orbits such that one obtains

Lemma 3.4 In a generic family of vector fields the intersection of $W_{\lambda}^{c s}$ and $W_{\lambda}^{c u}$ in $\Sigma$ consists of two curves $\mathfrak{C}_{\lambda} \subset$ Fix $(R)$ and $\mathfrak{D}_{\lambda} \subset$ Fix $(Q)$.

Again performing a projection along stable fibres we obtain two curves in $W_{\lambda}^{c}$ for which we assume an equivalent condition to (H6) by 


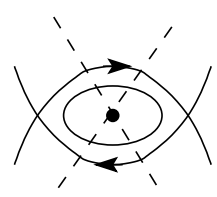

$\lambda_{1}<0$

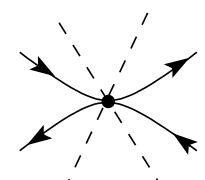

$\lambda_{1}=0$

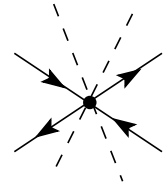

$\lambda_{1}>0$

Figure 9. Bifurcation diagram for one-homoclinic orbits near $\Gamma \subset \operatorname{Fix}(S)$ in case of the normal form (5). The dashed lines show the curves $\mathfrak{C}_{\lambda}, \mathfrak{D}_{\lambda}$.
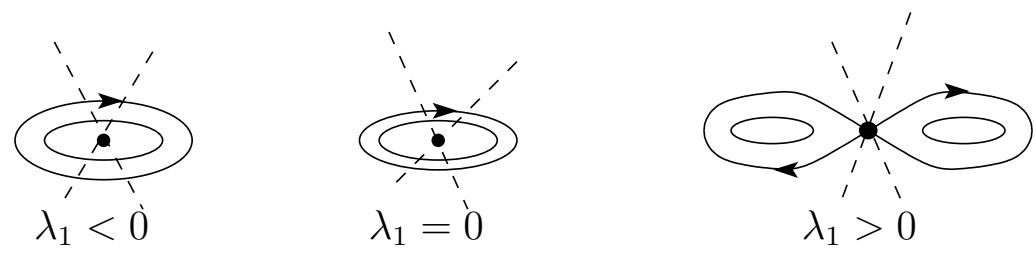

Figure 10. Bifurcation diagram for one-homoclinic orbits near $\Gamma \subset \operatorname{Fix}(S)$ in case of the normal form (6). The dashed lines show the curves $\mathfrak{C}_{\lambda}, \mathfrak{D}_{\lambda}$.

(H11) $\mathfrak{C}_{\lambda=0} \pitchfork \operatorname{Fix}(R), \mathfrak{D}_{\lambda=0} \pitchfork \operatorname{Fix}(R)$.

One then finds the bifurcation diagrams in Figures 9 and 10. Note, that assumption (H12) prevents any intersections of $\mathfrak{C}_{\lambda}, \mathfrak{D}_{\lambda}$ with the local stable manifold of 0 in $W_{\lambda}^{c}$. We therefore obtain

Theorem 3.5 Consider (1) under (H1)-(H3), (H10), and (H11) with parameter $\lambda \in \mathbb{R}$. Then for all $\lambda$ there exists a fast decaying homoclinic orbit $\Gamma \subset$ Fix $(S)$. Generically, there are no other one-homoclinic orbits to the origin.

Remark. The studies in [32] are more comprehensive in that they give a complete description of bifurcating one-homoclinic orbits to the centre manifolds $W_{\lambda}^{c}$. In that paper particular emphasis is given to the case where the local bifurcation is governed by the normal form (5). It is proved that the local scenario is then accompanied by a similar global bifurcation, creating two homoclinic orbits to the equilibria that emerge in the local bifurcation. This has been termed a reversible homoclinic pitchfork bifurcation in [32]. But as explained before, this bifurcation is not of concern for us here since we concentrate on homoclinic orbits to the origin in the present paper. 


\section{Reversible Hamiltonian systems}

We now turn to the important class of systems that are both reversible and Hamiltonian. As discussed in the beginning of the paper most of the physical applications of our studies lead to an analysis of such equations (compare also with the examples in Section 5 below). We will show that the previously obtained results are in this case still valid for symmetric solutions although as we shall see the basic transversality condition (H4) is not satisfied. The main emphasis will be laid on the general case in Section 2, the implications for systems with additional $\mathbb{Z}_{2}$-symmetry will be discussed only shortly.

So let us consider (1) under assumptions (H1)-(H3) and demand in addition that

(H13) There exists a function $H: \mathbb{R}^{4} \times \mathbb{R}^{2} \rightarrow \mathbb{R}$ such that (1) can be written as

$$
\dot{x}=J \cdot \nabla H(x, \lambda),
$$

where

$$
J=\left(\begin{array}{rr}
0 & I \\
-I & 0
\end{array}\right)
$$

denotes the standard symplectic structure on $\mathbb{R}^{4}$.

A well-known property of (7) is that the Hamiltonian $H$ is a first integral for the equation, i.e. it is constant along orbits of the system. It is this conservative character of the equation which is of relevance for the following analysis. We also note that the reversibility of (7) is reflected by the fact that $H \circ R=H$.

Proceeding as usual we find that the local bifurcation of 0 in $W_{\lambda}^{c}$ is described by the normal form (2). The determination of homoclinic orbits to $W_{\lambda}^{c}$, however, is difficult. Similar to the last section the property of (7) being Hamiltonian imposes additional restrictions for $W_{\lambda}^{c s(c u)}$ and does not allow our basic assumption (H4) to be fulfilled. To see this note that the cross-section $\Sigma$ is smoothly foliated by level-sets $\mathcal{H}_{h}$ of the Hamiltonian $H$. Each of these intersects the space Fix $(R)$ transversally and with no loss of generality we can assume them to be straightened out, i.e. $\mathcal{H}_{h} \subset T \mathcal{H}_{0}$. (Here we assume that the equilibrium is contained in the zero level set $\mathcal{H}_{0}$ of $H$.) In addition, Lemma 2.2 implies that for $\lambda_{1}<0, \lambda_{2}=0$ there exists a homoclinic orbit to the saddlecentre 0 . Let us consider the consequences. Reduced to the centre manifold the Hamiltonian $H$ has a local extremum, say minimum. If $H(0)=0$ this implies that all points in $W^{c s(c u)}$ take non-negative values of $H$ which in turn implies that the traces of $W^{c s(c u)}$ in $\Sigma$ lie "on one side" of the trace of $\mathcal{H}_{0}$ in $\Sigma$. This observation shows that the tangent spaces of both manifolds at $\gamma(0)$ 
(a)

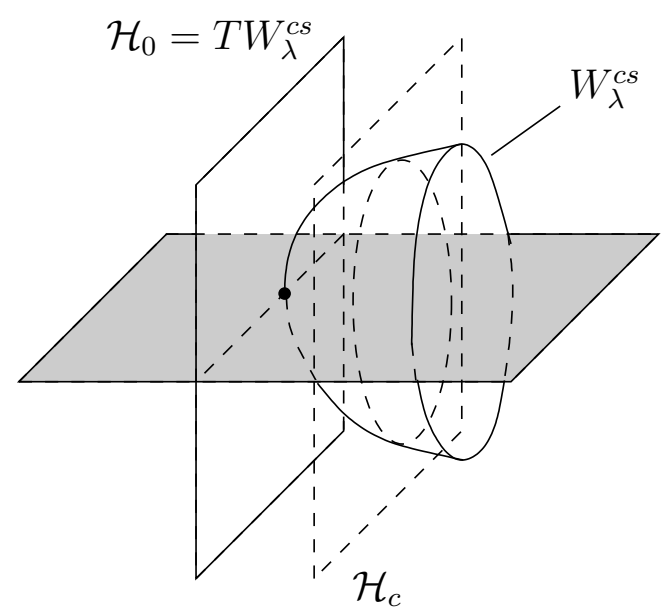

(b)

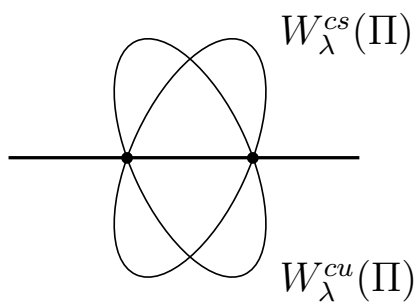

Figure 11. Situation in $\Sigma$ in the Hamiltonian case: For parameter values where a homoclinic orbit to a saddle-centre equilibrium exists the trace of $W_{\lambda}^{c s}$ is tangent to the zero level set $\mathcal{H}_{0}$ of the Hamiltonian and intersects Fix $(R)$ transversally. (By reversibility the same statement is valid for $W_{\lambda}^{c u}$.) Panel (a) of the picture shows a sketch of the intersection the stable and unstable manifold of a periodic orbit $\Pi$ in some level set $\mathcal{H}_{c}$ of $H$ in $\Sigma$. Note that in addition to the intersection points in Fix $(R)$ these manifolds will generically intersect in two further points giving rise to a pair of non-symmetric solutions as in (b).

are contained in the tangent space of $\mathcal{H}_{0}$; see [24] for a rigorous proof and Figure 11 for an illustration. Clearly, the same relations must be found for $\lambda=0$ such that we obtain

Lemma 4.1 Consider ( ( 7$)$ under (H1)-(H3). Then $T_{\gamma(0)} W_{\lambda=0}^{c s}=T_{\gamma(0)} W_{\lambda=0}^{c u}$ and $T_{\gamma(0)} W_{\lambda=0}^{c s} \pitchfork \operatorname{Fix}(R)$.

Because of the non-transverse intersection of $W_{\lambda=0}^{c s}$ and $W_{\lambda=0}^{c u}$ we expect both $R$-symmetric and non-symmetric solutions to bifurcate from $\Gamma$, compare again with Figure 11. In the following we concentrate on the bifurcation of symmetric orbits. Being more involved, a complete analysis of the bifurcation including the discussion of non-symmetric solutions is the subject of future research.

The existence of symmetric homoclinic orbits can be discussed in the same way as before. Lemma 4.1 implies the existence of a manifold of homoclinic orbits to $W_{\lambda}^{c}$ which intersects the cross-section $\Sigma$ in some curve $\mathfrak{D}_{\lambda}$. Hence, we are in precisely the same situation as in Section 2.2 and immediately obtain the final theorem.

Theorem 4.2 Consider the Hamiltonian system (7) under assumptions (H1)- 
(H3), (H5), and (H6). Then the existence of R-symmetric homoclinic orbits near $\Gamma$ is described by Theorem 2.3.

Remark. In the same way one can discuss the different cases in Section 3 under the additional hypotheses that the systems are Hamiltonian. One then finds that the main theorems in the corresponding sections describe the existence of symmetric homoclinic orbits near the primary orbit $\Gamma$.

\section{Three numerical examples}

We shall illustrate our theoretical results with numerical computations for three Hamiltonian example systems. First, we deal with a reversible fourthorder equation arising in a water-wave problem which is part of the general family of 5th-order $\mathrm{KdV}$ models. Afterwards, we study two problems from nonlinear optics involving reversible and $\mathbb{Z}_{2}$-symmetric systems.

For the numerical investigations we have used the methods for homoclinic continuation implemented in AUTO/HomCont, [14].

\subsection{A fifth-order KdV equation}

In this section we will illustrate the results of Section 2 with numerical studies for a fourth-order equation arising in water-wave theory. We are concerned with the existence of solitary wave solutions for the following fifth-order longwave equation for gravity-capillary water waves,

$$
r_{\tau}+\frac{2}{15} r_{x x x x x}-b r_{x x x}+3 r r_{x}+2 r_{x} r_{x x}+r r_{x x x}=0 .
$$

Which is an example of a general family of 5th-order $\mathrm{KdV}$ equations, see [20,33]. Making the travelling wave ansatz $r(t=x-a \tau)$ and integrating the resulting ODE once, we obtain the fourth-order equation

$$
\frac{2}{15} r^{i v}-b r^{\prime \prime}+a r+\frac{3}{2} r^{2}-\frac{1}{2}\left(r^{\prime}\right)^{2}+\left[r r^{\prime}\right]^{\prime}=0,
$$

where a prime denotes differentiation with respect to $t$. Homoclinic orbits to the origin of (8) were intensively studied in [6], using a combination of analytical and numerical techniques.

Here we are interested in the situation for $a=0$ and $b=2$ since for these parameter values the fundamental assumptions (H1)-(H3) are fulfilled. First 
note, that for all parameter values $a, b$ equation (8) is reversible with respect to

$$
R:\left(r, r^{\prime}, r^{\prime \prime}, r^{\prime \prime \prime}\right) \mapsto\left(r,-r^{\prime}, r^{\prime \prime},-r^{\prime \prime \prime}\right) .
$$

Note also, that one can introduce variables such that (8) may be written as a Hamiltonian system; see [6] for details. Moreover, the origin is an equilibrium for all $a, b$ which is easily seen to possess a double non-semisimple eigenvalue 0 and a pair of real eigenvalues if $a=0, b>0$. Increasing the parameter $a$ through 0 , the equilibrium undergoes a transcritical bifurcation described by the normal form (2). The equilibrium turns from a saddle-centre $(a<0)$ into a real saddle $(a>0)$. In particular, for $a>0$ a small homoclinic orbit emerges which corresponds to the famous Korteweg-de Vries solitary wave (after an appropriate rescaling of the system).

Finally, in [6] the following explicit homoclinic solution

$$
r_{h}(t)=3\left(b+\frac{1}{2}\right) \operatorname{sech}^{2}\left(\frac{\sqrt{3}}{2}(2 b+1) t\right)
$$

of $(8)$ is found for parameter values $a=3 / 5 \cdot(2 b+1)(b-2), b \geq-1 / 2$. In particular, $r_{h}$ exists for $a=0$ and $b=2$ where it is a fast decaying homoclinic solution. So the results of Sections 2 and 4 should apply to this situation.

In fact, numerical studies in [6] already revealed the orbit-flip bifurcation of the primary homoclinic orbit for $a>0$. Also the existence of the curve $L$ where the saddle-node bifurcation of homoclinic orbits occurs could be numerically verified. This curve can be approximated by taking different values of $a>0, b$ for which $r_{h}$ exists and performing a numerical continuation of the homoclinic solution with decreasing $b$ and fixed $a$. For example, for $a=0.1$ we can continue the starting solution with decreasing $b$ until we find a limit point at $b^{*}=1.83226$. In Figure 12 we present the bifurcation diagram together with corresponding plots of solutions at the indicated points. We have found comparable results for all tested parameter values.

The results in Figure 12 show excellent agreement with the general theory. Indeed, we find that in the reversible orbit-flip bifurcation two homoclinic orbits emerge, of which one is composed of the fast decaying solution and the (small) 'KdV'-homoclinic orbit, see panel b) in Figure 12. The two homoclinic orbits coalesce in a saddle-node bifurcation, and we find that the corresponding bifurcation curve $L$ is essentially linear.

Also the existence of an algebraically decaying solution for $a=0, b>2$ can be verified numerically. In Figure 12 such a solution is shown for parameter values $a=0, b=2.5$. Another illustration is given in Figure 13 where we compare this solution to the exponentially decaying solution at $a=0.1, b=2.5$. The plots in part c) of this figure indicate that the solution for $a=0$ decays with a quadratic rate in accordance to results of Section 2 . 


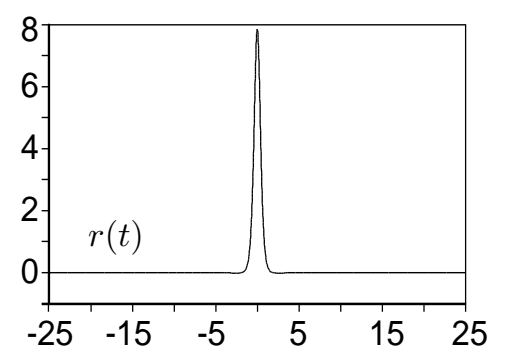

(a) $b=2.05, a=0.5$

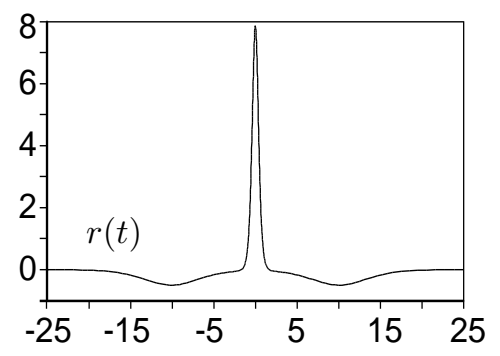

(b) $b=2.05, a=0.5$

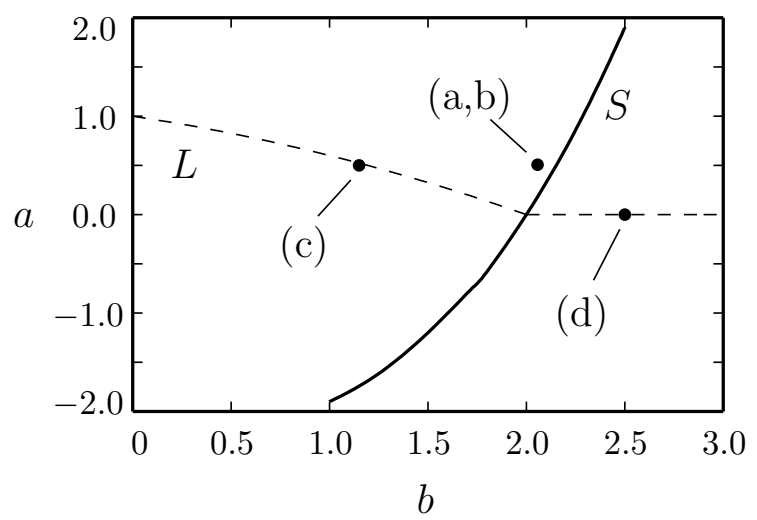

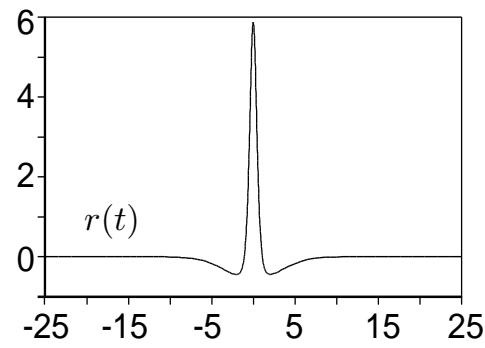

(c) $b=1.13, a=0.5$

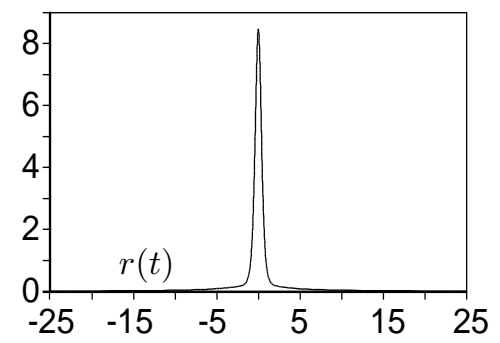

(d) $b=2.5, a=0.0$

Figure 12. Bifurcation diagram for (8) as computed with AUTO. On the solid curve $S$ the analytically known solution $r_{h}$ exists. For $a>0$ this curve describes a reversible orbit-flip. After decreasing $b$ two homoclinic solutions can be found. In a) the slow decaying solution after the orbit-flip is shown. In b) we show a plot of the second solution which is composed of the primary orbit and the small ' $\mathrm{KdV}$ ' homoclinic solution. These two orbits coalesce in a saddle-node bifurcation on the curve $L$ to the left of $S$ (see panel c)). For parameter values $b>2, a=0$, represented by the dashed line to the right of $S$, computations with AUTO show the existence of an algebraically decaying homoclinic solution as depicted in d) see also Figure 13.

So, for equation (8) we can compute everything that was predicted by the theory. We remark that for this example we have not made use of the reversible symmetry of the equation (which is possible in AUTO). Nevertheless, all solutions found have been symmetric under time-reversal which suggests that only symmetric one-homoclinic solutions bifurcate from the primary homoclinic orbit. This is of interest since the results in Section 4 did not concern 
(a)

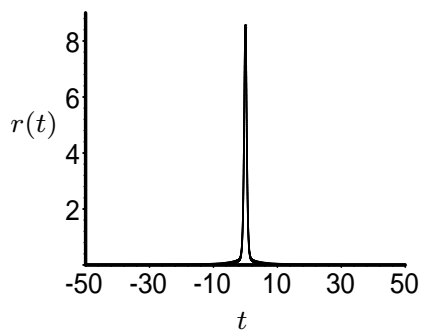

(b)

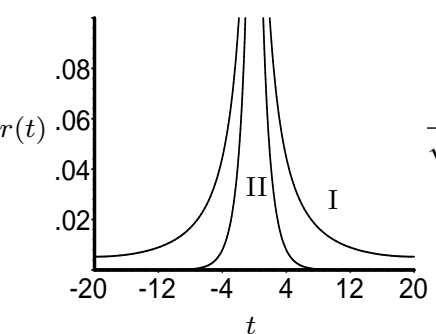

(c)

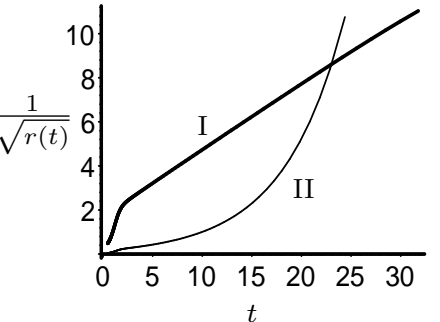

Figure 13. Comparison of the solutions for $a=0, b=2.5$, (I), and $a=0.1, b=2.5$, (II). Panels (a) and (b) show plots of the solutions. In (c) the reciprocal of the square-root of the solutions is plotted. Here the linearity of (I) as $t \rightarrow \infty$ reveals the quadratic rate of decay for the solution whereas (II) decays at some higher (exponential) rate. Note that the solution (II) in (c) is multiplied by a factor $1 / 10$ in order to view it on the same set of axes.

non-symmetric solutions.

Note that we do not attempt to prove any of the results for (8) rigorously. This would amount to proving that the equation fulfills the non-degeneracy conditions imposed for the general analysis. In particular regarding (H6) this is a major difficulty. But since we find perfect agreement of the numerical and the theoretical results we could argue the other way around and claim that (8) is a generic system in the sense of this paper. So our analysis can explain the numerical results obtained for the equation.

\subsection{Two examples from nonlinear optics}

In connection with the general results of Section 3.1 and 3.2 we study two examples which additionally possess $\mathbb{Z}_{2}$-symmetry. Both deal with the existence of embedded solitons in nonlinear optical media. An example for the class of systems studied in Section 3.3 can be found [32] where also some numerical results are presented.

\subsubsection{An extended massive Thirring model}

We first consider an extended massive Thirring model that describes solitons in an optical media equipped with Bragg-grating, see [9] and references therein. The model is described by the following systems of complex PDEs

$$
\begin{array}{r}
i u_{t}+i u_{x}+D u_{x x}+\left(\sigma|u|^{2}+|v|^{2}\right) u+v=0 \\
i v_{t}-i v_{x}+D v_{x x}+\left(\sigma|v|^{2}+|u|^{2}\right) v+u=0 .
\end{array}
$$


Looking for steady state solutions via $u(x, t)=e^{i \chi t} U(x), v(x, t)=e^{-i \chi t} U(x)$ we can perform scalings and assume with no loss of generality that $\sigma=0$ and $U=V^{*}$, where $V^{*}$ denotes the complex conjugate of $V$. Doing so, we obtain the single complex ODE

$$
D U^{\prime \prime}+i U^{\prime}+\chi U+U|U|^{2}+U^{*}=0,
$$

which is reversible with respect to

$$
R:\left(U, U^{\prime}\right) \mapsto\left(U^{*},-\left(U^{\prime}\right)^{*}\right)
$$

and has odd symmetry

$$
S:\left(U, U^{\prime}\right) \mapsto-\left(U, U^{\prime}\right)
$$

We note again that one can write (9) as a Hamiltonian system in $\mathbb{R}^{4}$.

For $|\chi|<1$ the origin is a saddle-centre equilibrium of (9) and at $\chi=-1$ it undergoes a reversible pitchfork bifurcation of figure-eight-type to become a real saddle itself.

In [10] it was found numerically that there are three curves in the $(D, \chi)$ plane at which embedded solitons exist. Each of these curves can be extended to parameter values $\chi<-1$, so that there exist three points in the parameter plane around which the results of Section 3.1 apply, see Figure 14. (Note that because of the odd symmetry of (9) embedded solitons, i.e. homoclinic orbits, come in pairs. But obviously it suffices to consider one of the two orbits for our purposes.) We restrict attention to one of the points and choose the point $\left(D^{*}, \chi^{*}\right)=(1.5,-1)$ with the largest $D$-value for our computations.

In accordance with Theorem 3.1 we can detect a reversible orbit-flip bifurcation of the primary homoclinic orbit and two curves where a saddle-node bifurcation of homoclinic orbits occur. In Figure 15 the bifurcation diagram including plots of the real parts of the corresponding homoclinic solutions is shown. In the computations we have explicitly used the reversing symmetry of (9). As for example (8) above we find that the reversible orbit-flip bifurcation of the primary orbit also gives rise to a solution which is composed of the fast decaying solution and a small homoclinic solution in the centre manifold. Note, however, that for (9) there are two different small homoclinic solutions and which one is chosen depends on whether $D$ is decreased or increased.

We remark that for this example the computations have to be performed very close to the critical parameter values to find agreement with the general bifurcation results concerning the shape of the "saddle-node curves". On the other hand, in order to illustrate the different types of bifurcating solutions it is necessary to compute the solutions in Figure 15 for larger parameter values. 


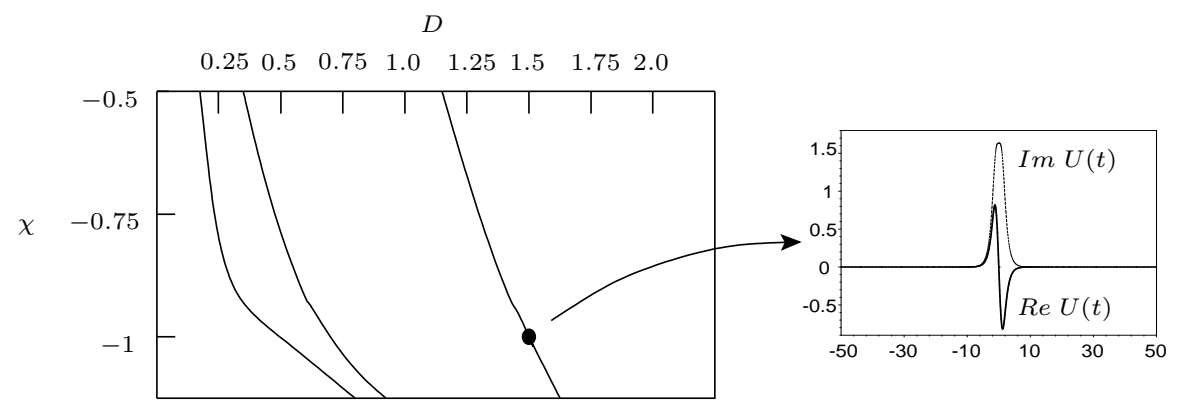

Figure 14. The three curves in the $(D, \chi)$-parameter plane at which homoclinic solutions of (9) were computed in [10] to cross the boundary of the region for which the origin is a saddle-centre $(|\chi|<1)$. The indicated solution at $\left(D^{*}, \chi^{*}\right):=(1.5,-1)$ is the one whose unfolding is computed in Figure 15.

This explains why the bifurcation diagram in this figure is only schematic for $\chi<-1.00005$.

\subsubsection{A second-harmonic-generation system}

We end this section with computations for the system in connection to which the term embedded solitons was used first. In [34] solitary waves appearing in an optical medium with competing quadratic and cubic nonlinearities were studied. The model is given by

$$
\begin{aligned}
& i u_{z}+\frac{1}{2} u_{t t}+u^{*} v+\gamma_{1}\left(|u|^{2}+2|v|^{2}\right) u=0 \\
& i v_{z}+\frac{1}{2} \delta v_{t t}+q v+\frac{1}{2} u^{2}+2 \gamma_{2}\left(|v|^{2}+2|u|^{2}\right) v=0
\end{aligned}
$$

where $u$ and $v$ are the amplitudes of complex wave vectors corresponding to the fundamental and second-harmonic fields. Seeking stationary solutions in the form $u=U(t) \exp (i k z), v=V(t) \exp (2 i k z)$, with real $k$ we are led to the system of ODEs for $U, V$

$$
\begin{aligned}
& \frac{1}{2} U^{\prime \prime}-k U+U V+\gamma_{1}\left(|U|^{2}+2|V|^{2}\right) U=0 \\
& -\frac{1}{2} \delta V^{\prime \prime}+(q-2 k) V+\frac{1}{2} U^{2}+2 \gamma_{2}\left(|V|^{2}+2|U|^{2}\right) V=0 .
\end{aligned}
$$

Embedded soliton solution of (10) have been found for $\delta>0$ and $\gamma_{1,2}<0$ in [34], and for $\delta<0, \gamma_{1,2}>0$ in [11]. We shall follow the latter paper here and search for parameter values $\delta<0$ where our theory applies. In the following we will fix the parameters $\gamma_{1}=\gamma_{2}=0.05, k=1$ and consider (10) as a system depending on the two parameters $\delta, q$.

Let us first discuss the symmetries of (10). The system is reversible with 


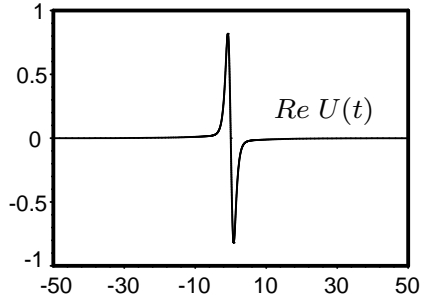

(a) $D=1.504$

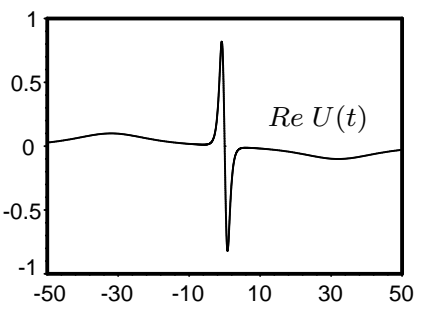

(b) $D=1.504$

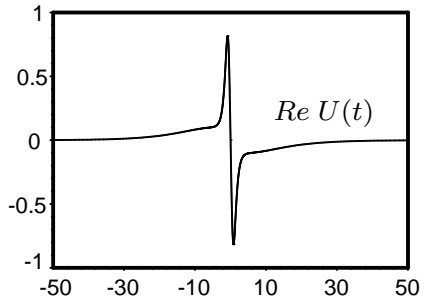

(c) $D=1.488$

$D$

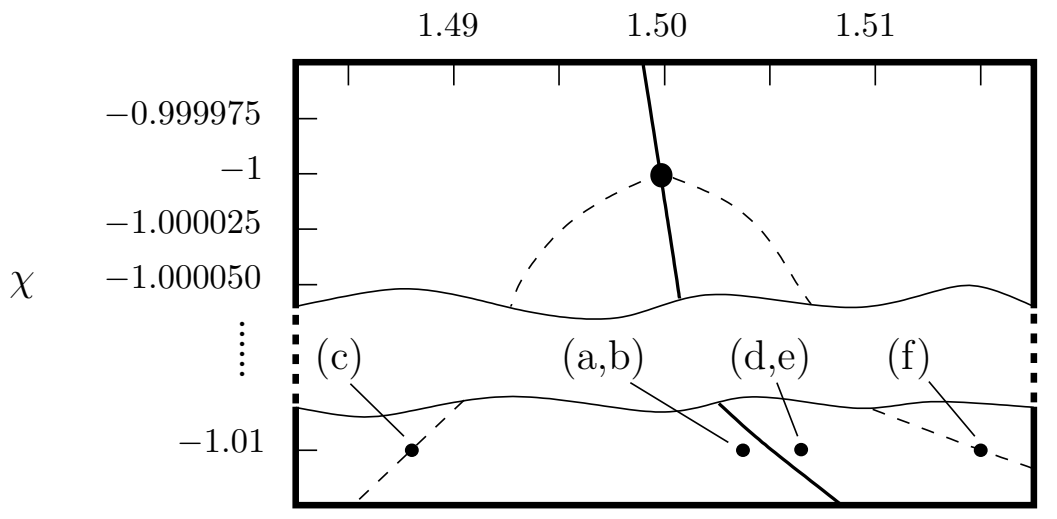

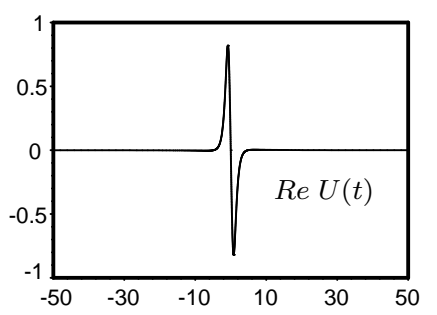

(d) $D=1.506$

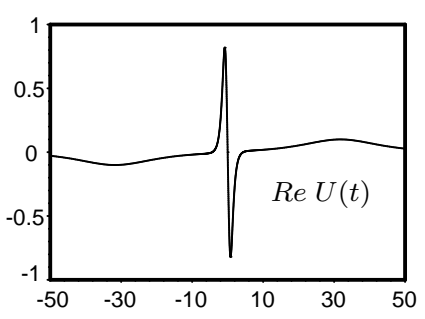

(e) $D=1.506$

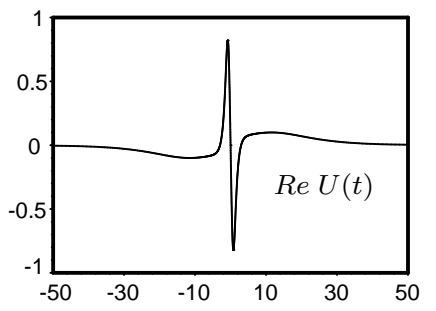

(f) $D=1.515$

Figure 15. Bifurcation of one-homoclinic orbits of $(9)$ near $\left(D^{*}, \chi^{*}\right)$, computed using AUTO. On the solid curve, the fast decaying homoclinic orbit exists and for $\chi<-1$ this defines an orbit-flip bifurcation. On the dashed curves saddle-node bifurcations of homoclinic orbits were detected. The solutions in the sub-panels were all computed for $\chi=-1.01$ and the given values of $D$. Only the real parts of the solutions are shown.

respect to

$$
R:\left(U, U^{\prime}, V, V^{\prime}\right) \mapsto\left(U,-U^{\prime}, V,-V^{\prime}\right)
$$

and $\mathbb{Z}_{2}$-symmetric with respect to

$$
S:\left(U, U^{\prime}, V, V^{\prime}\right) \mapsto\left(-U,-U^{\prime}, V, V^{\prime}\right)
$$

The origin is an equilibrium for all parameter-values. We are interested in the 
situation at $q=2$ since on this line the equilibrium has a zero eigenvalue and a pair of real eigenvalues. Moreover, it is easy to compute that the centre eigenspace of the linearisation at this equilibrium is contained in Fix $(S)$. According to Section 3.2 we would therefore expect a transcritical bifurcation of the equilibrium. Equation (10), however, is non-generic since we find that the local bifurcation is governed by the normal form (6). This means that the equilibrium undergoes a pitchfork bifurcation of figure-eight type. Nevertheless, our general studies still apply to this system. As it was observed after Corollary 3.2 we just have to adapt the results of Section 3.1 in this case.

In [11] curves (in the $(\delta, q)$-parameter plane) of $R$-symmetric embedded soliton solutions were found for $k=0.3$. In a similar manner we find a curve of such solutions for $k=1$. This curve can be extended to parameter values $q<2$ where the origin is a real saddle, see Figure 16. The critical parameter value is given by $\left(\delta^{*}, q^{*}\right)=(-2.6425,2)$. Now we can go again through computations similar to the last example. The results are comprised in the bifurcation diagram in Figure 16. We have again incorporated plots of solutions of (10) for several parameter values. For the purpose of illustrating the different types of homoclinic solutions we only show the $V$-component of the solutions.

\section{Discussion}

Our goal in this paper has been to analyse the nature of solution profiles and parameter dependency of solitary wave solutions to nonlinear PDE systems in $1+1$ dimensions that undergo a transition from being a gap soliton to an embedded soliton. Using dynamical systems theory this was formulated as a bifurcation problem concerning a homoclinic orbit asymptotic to a degenerate equilibrium. We studied different scenarios for the bifurcation of onehomoclinic orbits from the primary one in certain classes of reversible systems including also effects of $\mathbb{Z}_{2}$-symmetry and Hamiltonian structure. The analytical results were shown to match numerical experiments on model-systems arising in nonlinear optics and water wave theory.

It is clear that this paper only takes a first step towards a complete understanding of the homoclinic bifurcation and the underlying physical problem. A natural next issue for investigations is the bifurcation of $n$-homoclinic orbits corresponding to $N$-pulse solutions of the physical problem. Some of our results already give rise to interesting questions. For example, general results by Sandstede et. al. in [30] show that in reversible systems the orbit-flip bifurcation which was detected in all cases of Sections 2 and 3 generically leads to the emergence of $n$-homoclinic orbits. See also [6] for a numerical investigation of this bifurcation for the 5 -th order $\mathrm{KdV}$-equation (8), where the presence of a Hamiltonian structure implies that the bifurcation is degenerate compared 


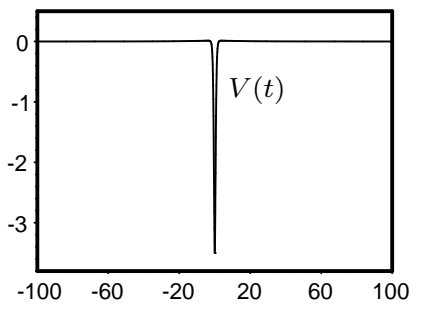

(a) $\delta=-2.66$

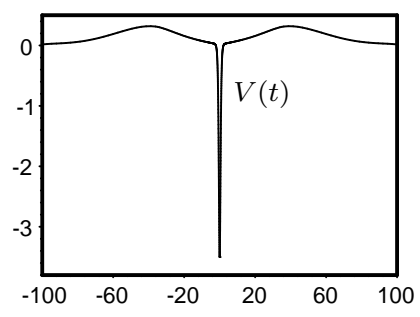

(b) $\delta=-2.66$

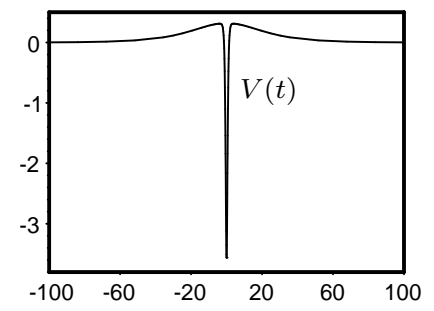

(c) $\delta=-3.03$

$\delta$

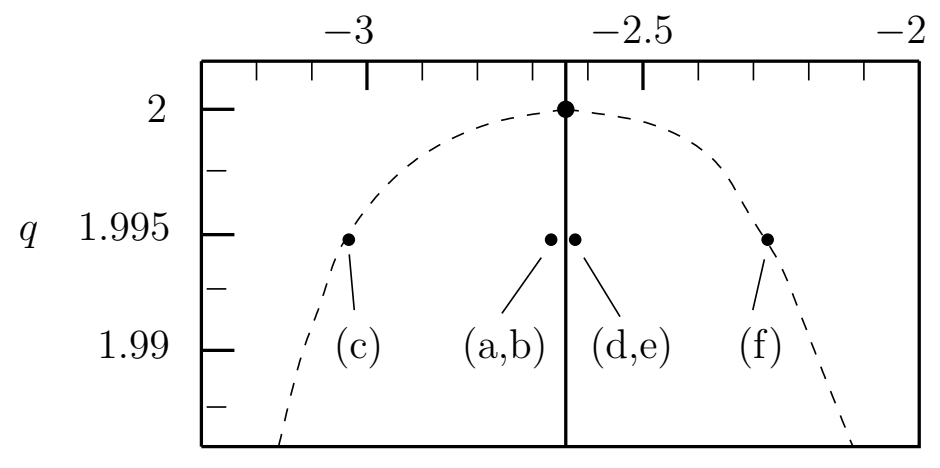

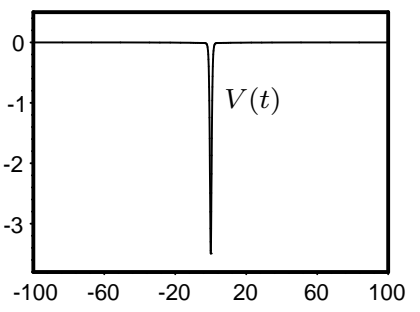

(d) $\delta=-2.63$

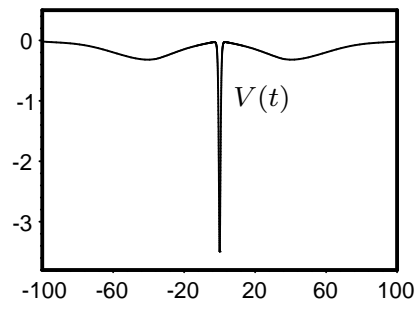

(e) $\delta=-2.63$

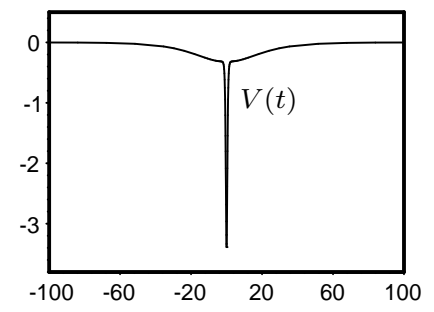

(f) $\delta=-2.28$

Figure 16. Bifurcation diagram for one-homoclinic orbits of $(10)$ near $\left(\delta^{*}, q^{*}\right)$. The fast decaying homoclinic orbit exists at the solid curve whereas at the dashed curves the saddle-node bifurcation of homoclinic orbits occurs. The corresponding solutions have been computed for $q=1.995$ and the given values of $\delta$. Plots show the $V$-components of the solutions.

with the analysis of [30]. These results concern orbits which are composed of copies of the fast decaying homoclinic orbit alone. In our case, however, there is the possibility of an even richer dynamics in that it includes orbits being composed of copies of the primary orbit and of parts that are governed by the dynamics in $W_{\lambda}^{c}$. Such a solution can for instance be found in panel b) of Figure 12.

Of similar interest is the existence of $N$-pulse solutions for parameter values where the origin is a saddle-centre. Here, general results are available which 
explain the accumulation of such solutions on parameter values where the primary orbit exists, see [7] for the reversible case and [27,23] for the case of systems that are additionally Hamiltonian. An interesting point is that these studies show differences in the behaviour of systems that are purely reversible and of those are also Hamiltonian. In Section 4 of the present paper we have established a possible reason for these differences, namely the fact that the Hamiltonian property does not allow a transverse intersection of the centre-stable and unstable manifolds. This will certainly be reflected in results concerning bifurcating $n$-homoclinic orbits. Thus, our geometric approach to the analysis may also give further insight into qualitative differences between reversible and Hamiltonian systems, see [7] for some related remarks.

From a PDE point of view a further important project is the study of the stability of solutions. More precisely an open question is what happens to the stability of the ESs when they cross the critical parameter value to become structurally stable objects. For a variety of model equations, including two of the examples studied in this paper, the property of semi-stability of embedded solitons has been established by a mixture of numerical, asymptotic and rigorous arguments $[28,33,34,35]$. Does this semi-stability necessarily transform into true exponential stability when the embedded soliton becomes a gap soliton? A general rigorous answer to this question is of course beyond the realm of the finite-dimensional dynamical systems theory used in this paper.

Acknowledgments. This paper was written when T. W. visited the University of Bristol in 2001/02, supported by a DAAD grant. He thanks the University of Bristol for the warm hospitality. A.R.C. acknowledges support of the U.K. EPSRC, with whom he holds an advanced fellowship.

\section{References}

[1] J. P. Boyd. Weakly Nonlocal Solitary Waves and Beyond-All-Orders Asymptotics. Kluwer, Dodrecht, Boston, London, 1998.

[2] B. Buffoni, A. R. Champneys, and J. F. Toland. Bifurcation and coalescence of a plethora of homoclinic orbits for a Hamiltonian system. J. Dyn. Diff. Eqns., 8:221-281, 1996.

[3] A. V. Buryak. Stationary soliton bound states existing in resonance with linear waves. Physical Review E, 52:1156-1163, 1995.

[4] D. C. Calvo and T. R Akylas. On the formation of bound states by interacting nonlocal solitary waves. Physica D, 101:270-288, 1997.

[5] A. R. Champneys. Homoclinic orbits in reversible systems and their applications in mechanics, fluids and optics. Physica D, 112:158-186, 1998. 
[6] A. R. Champneys and M. D. Groves. A global investigation of solitary wave solutions to a two-parameter model for water waves. J. Fluid Mech., 342:199229, 1997.

[7] A. R. Champneys and J. Härterich. Cascades of homoclinic orbits to a saddlecentre for reversible and perturbed hamiltonian systems. Dynamical Systems, 15:231-252, 2000.

[8] A. R. Champneys and B. A. Malomed. Embedded solitons in a three-wave system. Phys. Rev. E, 61:463-466, 1999.

[9] A. R. Champneys and B. A. Malomed. Moving embedded solitons. Phys. Rev. A, 32:547-553, 1999.

[10] A. R. Champneys, B. A. Malomed, and M. J. Friedman. Thirring solitons in the presence of dispersion. Phys. Rev. Lett., 80:4168-4171, 1998.

[11] A. R. Champneys, B. A. Malomed, J. Yang, and D. J. Kaup. Embedded solitons: solitary waves in resonance with the linear spectrum. Physica D, 152:340-354, 2001.

[12] C. M. de Sterke and J. E. Sipe. Gap solitons. Progress in Optics, 33:203-260, 1993.

[13] R. L. Devaney. Reversible diffeomorphisms and flows. Trans. Amer. Math. Soc., 218:89-113, 1976.

[14] E. J. Doedel, A. R. Champneys, T. R. Fairgrieve, Yu. A. Kuznetsov, B. Sandstede, and X. J. Wang. AUTO97 continuation and bifurcation software for ordinary differential equations, 1997. Available by anonymous ftp from FTP.CS.CONCORDIA.CA, directory PUB/DOEDEL/AUTO.

[15] F. Dumortier. Local study of planar vector fields: singularities and their unfoldings. Structures in Dynamics, Studies in Mathematical Physics, 2:161$242,1991$.

[16] J. Fujioka and A. Espinosz. Soliton-like solution of an extenced NLS equation existing in resonance with linear dispersive waves. Journal of the Physical Society of Japan, 66:2601-2607, 1997.

[17] R. Grimshaw and P. Cook. Solitary waves with oscillatory tails. In A. T. Chwang, J. H. W. Lee, D. Y. C. Leung, and A. A. Balkema, editors, Proceedings of Second International Conference on Hydrodynamics, Hong Kong 1996, pages 327-336. Hydrodynamics: Theory and Applications, volume 1, 1996.

[18] J. Guckenheimer and P. Holmes. Nonlinear Oscillations, Dynamical Systems and Bifurcations of Vector Fields. Springer-Verlag, New York, U.S.A., 1983.

[19] G. Iooss and Adelmeyer M. Topics in bifurcation theory and applications. World Scientific, 1992. Adv. Series in Nonlinear Dynamics 3.

[20] S. Kichenassamy and P. J. Olver. Existence and non-existence of solitary wave solutions to higher-order model evolution equations. SIAM J. Math. Anal., 23:1141-1166, 1996. 
[21] J. Klaus and J. Knobloch. Bifurcation of homoclinic orbits to a saddle-center in reversible systems. Int. Journal of Bifurcation of Chaos, to appear, 2002.

[22] J. Knobloch. Bifurcation of degenerate homoclinics in reversible and conservative systems. J. Dynamics. Diff. Eqns., 9:472-494, 1997.

[23] K. Kolossovski, A. R. Champneys, A. V. Buryak, and R. A. Sammut. Multipulse embedded solitons as bound states of quasi-solitons. Physica D, 171(3):153-177, 2002 .

[24] O.Yu Koltsova and L. M. Lerman. Periodic orbits and homoclinic orbits in a two-parameter unfolding of a Hamiltonian system with a homoclinic orbit to a saddle-center. Int. J. Bifurcation Chaos, 5:397-408, 1995.

[25] J. S. W. Lamb and Capel H. W. Local bifurcations on the plane with reversing point group symmetry. Chaos, Solitons and Fractals, 5:271-293, 1995.

[26] X.-B. Lin. Using Melnikov's method to solve Shil'nikov's problems. Proc. Roy. Soc. Edinburgh, A, 116:295-325, 1990.

[27] A. Mielke, P. Holmes, and O. O'Reilly. Cascades of homoclinic orbits to, and chaos near, a Hamiltonian saddle-center. J. Dynamics Diff. Eqns., 4:95-126, 1992.

[28] D. E. Pelinovsky and J. Yang. A normal form for nonlinear resonance of embedded solitons, 2002. to appear in Proc. Roy. Soc. Lond. A.

[29] B. Sandstede. Verzweigungstheorie homokliner Verdopplungen. PhD thesis, University of Stuttgart, 1993.

[30] B. Sandstede, C. K. R. T. Jones, and J. C. Alexander. Existence and stability of $N$-pulses on optical fibres with phase-sensitive amplifiers. Physica D, 106:167206, 1997.

[31] A. Vanderbauwhede and B. Fiedler. Homoclinic period blow-up in reversible and conservative systems. Z. Angew. Math. Phys., 43:292-318, 1992.

[32] T. Wagenknecht. About a homoclinic pitchfork bifurcation in reversible systems with additional $\mathbb{Z}_{2}$-symmetry. Nonlinearity, 15(6):2097-2120, 2002.

[33] J. Yang. Dynamics of embedded solitons in the extended KdV equations. Stud. Appl. Math., 106:337-365, 2001.

[34] J. Yang, B. A. Malomed, and Kaup D. J. Embedded-solitons in secondharmonic-generating systems. Phys. Rev. Lett., 83:1958-1961, 1999.

[35] J. Yang, B. A. Malomed, D. J. Kaup, and A. R. Champneys. Embedded solitons: a new type of solitary waves. Mathematic and Computers in Simulation, 56:585$600,2001$. 